\title{
NORMALIZATION FOR PLANAR STRING DIAGRAMS AND A QUADRATIC EQUIVALENCE ALGORITHM
}

\author{
ANTONIN DELPEUCH $^{a}$ AND JAMIE VICARY ${ }^{b}$ \\ ${ }^{a}$ Department of Computer Science, University of Oxford, Wolfson Building, Parks Road, Oxford, \\ OX1 3QD, UK \\ e-mail address: antonin.delpeuch@cs.ox.ac.uk \\ ${ }^{b}$ Computer Laboratory, University of Cambridge, J. J. Thomson Avenue, Cambridge, CB3 0FD, UK \\ e-mail address: jamie.vicary@cl.cam.ac.uk
}

\begin{abstract}
In the graphical calculus of planar string diagrams, equality is generated by exchange moves, which swap the heights of adjacent vertices. We show that left- and right-handed exchanges each give strongly normalizing rewrite strategies for connected string diagrams. We use this result to give a linear-time solution to the equivalence problem in the connected case, and a quadratic solution in the general case. We also give a stronger proof of the Joyal-Street coherence theorem, settling Selinger's conjecture on recumbent isotopy.
\end{abstract}

\section{INTRODUCTION}

0.1. Motivation and summary. String diagrams are a geometrical notation for the mathematical theory of monoidal categories, a logical toolkit for describing the algebra of compositional systems. Examples are given in Figure 1; a standard interpretation of such a diagram is that wires represent systems storing computational data, and vertices represent processes taking place over time (read from top to bottom), with each process having input and output data represented by the wires attached above and below the vertex respectively. Over the last 10 years, string diagrams have found increasingly broad application across theoretical computer science, in areas including quantum computation [Bac14, DL14, DDK10], natural language processing [CCS08], interacting agents [Mel12, GHWZ16], circuit design [GJL17], and rewriting [Mim14]; a key survey paper by Selinger [Sel11] has received over 400 citations in 10 years, with two-thirds of those in the last 5 years.

Despite this significant activity, there are no general results ${ }^{1}$ about the complexity of deciding equivalence of string diagrams, an important question if the theory is to become a mainstream logical technique that can form part of real-world systems. Equivalence of string diagrams is a geometrical notion, with two string diagrams being equivalent (that is,

This work was supported by an EPSRC scholarship.

${ }^{1}$ There are a variety of interesting results about the equivalence problem over some specific signatures, which we survey in Section 0.2.

DOI:10.46298/LMCS-18(1:10)2022
(C) A. Delpeuch and J. Vicary

(c) Creative Commons 
representing equal morphisms of the corresponding monoidal category) just when their string diagram representations are related by a recumbent isotopy [Sel11, Theorem 3, Caveat 9]. Here, isotopy means that one diagram can be deformed into the other without breaking, intersecting or reordering input or output wires, and recumbent means that inputs cannot exchange with outputs, and wires and vertices remain essentially 'upright' throughout the isotopy [JS91]. This equality relation is then sometimes collapsed further by adding additional axioms, in a way that suits the application.

In this paper we take a first look at the complexity of the general equivalence problem for planar string diagrams ${ }^{2}$ (henceforth simply diagrams), without additional axioms. This does not include all the features used by some applications of string diagrams (for example, braided or symmetric monoidal structure), but it is already a nontrivial setting, and seems a suitable place to begin building the theory.

Our main results are as follows. We write $v$ for the number of vertices in a diagram, and $e$ for the number of edges; also, we say that a diagram is connected when there is a path in the diagram between any two vertices, and boundary-connected when it is either connected, or every vertex has a path in the diagram to a boundary. See Figure 1 for examples of these notions.

- For boundary-connected diagrams, we build a rewrite strategy that generates the equality relation, show it is strongly normalizing (Theorem 2.14), show it terminates after $O\left(v^{3}\right)$ steps (Theorem 3.4), show the normal forms can be constructed in $O(v e)$ time (Theorem 5.8), and show equality can be decided in $O(v+e)$ time (Corollary 7.9).

- For general diagrams, with no constraints on connectivity, we use the above results to derive a scheme that decides equality in $O(v e)$ time (Theorem 6.3).

\footnotetext{
${ }^{2}$ By the work of Joyal and Street [JS91], this corresponds to the word problem for monoidal categories which are free on a given generating set of objects and morphisms. Furthermore, our results extend immediately to bicategories which are free on a given set of generating 1- and 2-morphisms, but we prefer to keep the discussion at the level of monoidal categories.
}

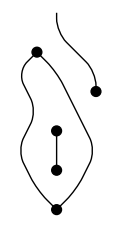

(A) Disconnected

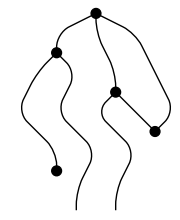

(B) Connected

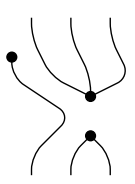

(c) Boundary-connected

Figure 1. Connectedness for string diagrams

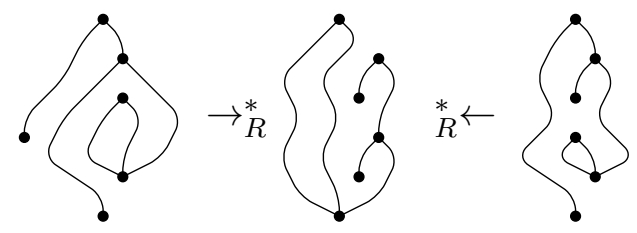

Figure 2. Two connected diagrams with the same right normal form 
- We show that the recumbency property listed above is unnecessary; that is, we show that two diagrams are recumbent isotopic, and hence equal, just when they are isotopic (Theorem 8.2). ${ }^{3}$ This proves a conjecture of Selinger [Sel11].

This final result is attractive, since in practice the recumbency property is highly constraining, forcing the entire diagram to remain essentially "vertical" throughout the isotopy.

0.2. Related work. The use of rewriting techniques on diagrams is ubiquitous in the communities which use monoidal or higher categories. Diagrammatic rewriting has been studied in detail for particular signatures, such as those of boolean circuits [Laf03, Laf09] or the ZX-calculus [DL14, DDK10]. More generally, rewriting theory of 2-polygraphs was developed by Guiraud and Malbos [GM18], extending classical results on monoids. In these approaches, the goal is to decide equality of diagrams up to the axioms in the signature, and structural equalities such as the exchange law or even symmetry are strict. Our results focus instead on the structural equalities, and do not allow equalities in the signature.

The word problem for the structural equalities has attracted attention in higher categories, but with no complexity result so far. The foundational work of Burroni [Bur93] establishes the link between the word problem for an algebraic structure and the path problem in the next dimension. Later, Makkai [Mak05] showed decidability of the word problem for higher cells in strict $\omega$-categories. Our work refines this result at dimension 2 by giving a complexity bound at dimension two. We also relate these computational results to the well established theory of embedded graphs (also called maps) [HW74, dd13], via reductions which bridge the differences in the notions of isotopy used.

The study of equivalence in category theory often takes the form of coherence results. These state that all morphisms between given source and targets and built from a particular signature are equal. These results often rely on rewriting techniques, the spirit of which was already present since Mac Lane's coherence theorem for monoidal categories [ML63]. More recently, Forest and Mimram [FM18] use rewriting to prove coherence for Gray monoids. They use similar techniques, with a focus on coherence of reductions rather than their length.

There has recently been activity in the development of computer proof assistants for string diagrams, including Quantomatic [DDK10], Globular [BKV16, BV] and its successor homotopy.io [HHV19]. Our string diagram isotopy algorithm could yield a geometrical notion of "tactic" for such a proof assistant, automatically finding isotopies between diagrams, or rearranging diagrams to normal form.

0.3. Outline. This paper has the following structure. We first introduce our formalism, defining diagrams and a rewriting relation on them. In Section 2, we show that the rewriting relation terminates on connected diagrams and derive an asymptotic upper bound on reduction length. Section 4 shows confluence of the rewriting relation, which gives a simple algorithm to normalize connected diagrams. Section 5 analyses the structure of right normal forms and shows how to compute them more efficiently. Section 6 extends these results to disconnected diagrams and Section 7 improves the complexity in the connected case by reducing the problem to the more widespread notion of planar map isotopy.

\footnotetext{
${ }^{3}$ This has a nice expression in categorical terms (Corollary 8.4): it says that for a monoidal signature $\Sigma$, the embedding functor from the free monoidal category on $\Sigma$ to the free pivotal category on $\Sigma$ is faithful.
} 


\section{Monoidal CATEgories AND StRing Diagrams}

We first introduce monoidal categories and show why morphism expressions written as terms are ill-suited to reason about equivalence. We then introduce string diagrams, which offer a more intuitive graphical representation of these morphisms, at the expense of requiring the manipulation of elaborate topological objects. Finally, we show how string diagrams can be encoded combinatorially, providing an efficient representation to detect equivalence.

\subsection{Monoidal categories.}

Definition 1.1. A monoidal category $\mathcal{C}$ is a category equiped with a bifunctor $\_\otimes_{-}: \mathcal{C} \times \mathcal{C} \rightarrow \mathcal{C}$ such that $\otimes$ is strictly associative and has a unit $I \in \mathcal{C}$.

In this work, the monoidal categories considered are strict, meaning that the associativity and unitality of the monoidal product are equalities rather than isomorphisms. The latter case corresponds to the notion of weak monoidal category. Mac Lane's coherence theorem states that any weak monoidal category is equivalent to a strict monoidal category.

Morphisms in monoidal categories can be composed in two ways. Given $f: A \rightarrow B$ and $g: B \rightarrow C$, we get $g \circ f: A \rightarrow C$ as in any category. This composition is associative and has units $1_{A}: A \rightarrow A$. This is intuitively a sequential composition, as $g$ is executed after $f$.

Given $u: A \rightarrow B$ and $v: C \rightarrow D$, we can use the bifunctor $\otimes$ to form $u \otimes v: A \otimes C \rightarrow$ $B \otimes D$. By interpreting the monoidal product $\otimes$ as a pairing operation, this is intuitively a parallel composition, as $u$ and $v$ are executed independly of each other, simultaneously.

The functoriality of $\otimes$ requires a compatibility between the two composition operations, called the exchange law or bifunctoriality equation:

$$
(g \otimes k) \circ(f \otimes j)=(g \circ f) \otimes(k \circ j)
$$

when both sides of the equation are defined.

The bifunctoriality equation above, combined with the associativity and unitality of $\otimes$ and $\circ$, gives rise to a rich equational theory. For instance, given morphisms $a, b: I \rightarrow I$, we have the following derivation, known as the Eckmann-Hilton argument:

$$
\begin{array}{rlrl}
a \circ b & =\left(a \otimes 1_{I}\right) \circ b & & \text { (unitality of } \otimes) \\
& =\left(a \otimes 1_{I}\right) \circ\left(1_{I} \otimes b\right) & & \text { (unitality of } \otimes) \\
& =\left(a \circ 1_{I}\right) \otimes\left(1_{I} \circ b\right) & & \text { (bifunctoriality) } \\
& =a \otimes\left(1_{I} \circ b\right) & & \text { (unitality of } \circ) \\
& =a \otimes b & & \text { (unitality of } \circ) \\
& =\left(1_{I} \circ a\right) \otimes b & \text { (unitality of } \circ) \\
& =\left(1_{I} \circ a\right) \otimes\left(b \circ 1_{I}\right) & \text { (unitality of } \circ) \\
& =\left(1_{I} \otimes b\right) \circ\left(a \otimes 1_{I}\right) & & \text { (bifunctoriality) } \\
& =b \circ\left(a \otimes 1_{I}\right) & & \text { (unitality of } \otimes) \\
& =b \circ a & & \text { (unitality of } \otimes)
\end{array}
$$

The derivation above shows multiple issues with the representation of morphisms as terms to reason about equivalence. First, the rewriting strategy used to derive the equality is not obvious: one needs to introduce identities by unitality in creative ways in steps $1,2,6$ 
and 7. Therefore it seems difficult to obtain a terminating and confluent rewriting system in this presentation. Second, the bifunctoriality equation only holds when the domains and codomains of the morphisms involved are compatible: one cannot, in general, replace any expression $(g \otimes k) \circ(f \otimes j)$ by $(g \circ f) \otimes(k \circ j)$. Thus we are required to keep track of the domains and codomains of all sub-expressions involved to understand which axiom can be applied. In the example above all domains and codomains are $I$ so the bifunctoriality equation could always be applied, but this is not true in general.

1.2. String diagrams. String diagrams are graphical representations of morphisms in a monoidal category. They were proposed independently by [Hot65] and [JS88, JS91].

Any term representing a morphism in a monoidal category can be inductively translated to a string diagram as shown in Figure 3. The reverse translation can be defined too. This first requires defining a class of well-behaved diagrams to ease the analysis: for instance, [JS91] require diagrams to be "recumbent":

Definition 1.2 [JS91]. A recumbent (or progressive) plane diagram is an embedded graph $\Gamma \hookrightarrow[a, b] \times \mathbb{R}$ (see [JS91]) such that the projection of any edge on the vertical axis is injective.

In the definition above, $\Gamma$ is a topological graph and $[a, b] \times \mathbb{R}$ is a section of the plane it gets embedded into. We can then associate to any recumbent string diagram a corresponding morphism in the free monoidal category on the generators involved. This is done by breaking down a diagram into basic blocks of either generators or identities and composing them sequentially or in parallel according to how these basic blocks are laid out. The requirement for edges to be injective along the vertical axis ensures that there are no "cups" or "caps" which would not be interpretable without adjoints. The bifunctoriality equation and the associativity and unitality of compositions ensure that the resulting morphism does not depend on the order in which the blocks are composed, as shown in Figure 4. The details of this construction can be found in [JS88, JS91].

The main result establishing the usefulness of string diagrams is the invariance of the interpretation as a morphism up to topological deformations.

Definition 1.3 [JS88, Definition 5]. A deformation of recumbent graphs is a deformation $h: \Gamma \times[0,1] \rightarrow[a, b] \times \mathbb{R}$ of planar graphs (see Definition 1.2) such that the image $\Gamma(t)$ of $h(-, t)$ is recumbent for all $t \in[0,1]$.

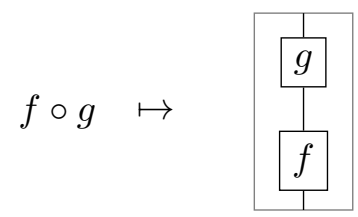

(A) Sequential composition

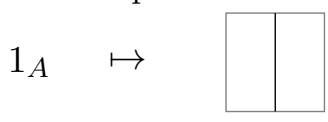

(c) Identity

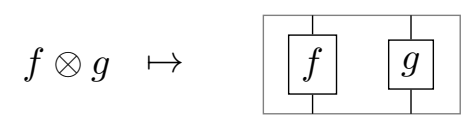

(B) Parallel composition

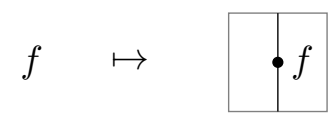

(D) Generator

FIGURE 3. Recursive translation of a morphism expression to a string diagram 
Theorem 1.4 [JS88, Theorem 3]. If $h: \Gamma \times[0,1] \rightarrow[a, b] \times \mathbb{R}$ is a deformation of recumbent diagrams then the value $v(\Gamma(t))$ is independent of $t \in[0,1]$.

This means that instead of manipulating terms to represent morphisms, we can simply rely on string diagrams to derive equalities in monoidal categories. For instance, our example derivation of $a \circ b=b \circ a$ for scalars is represented in string diagrams by Figure 5 . The argument can simply be understood as the rotation of two vertices around each other in the plane.

However, while this representation is easy to manipulate at an intuitive level, one can argue that the topological notions are relatively involved and describing precisely the objects involved is tedious. These graphical objects also seem harder to encode in a computer, making them of little use to solve the word problem.

In fact, it is possible to encode a string diagram more efficiently than by listing the explicit positions of its vertices and the trajectories of its edges. This relies on the notion of general position:

Definition 1.5. A string diagram is in general position when none of its vertices share the same height.

Any string diagram can be deformed slightly to be in a general position.

Lemma 1.6 [JS88]. Given a string diagram $\Gamma$, there exists a diagram $\Gamma^{\prime}$ in general position and a deformation of recumbent diagrams between $\Gamma$ and $\Gamma^{\prime}$.

Proof. If two vertices are at the same height, then they can be slightly perturbated such that one is above the other. This can be done for each pair of vertices with recumbent transformations.

Any string diagram in general position can be cut up in slices, each of which contains exactly one generator.

Lemma 1.7. Given a string diagram in general position $\Gamma$ containing $n$ vertices, there exists heights $h_{1}, \ldots, h_{n+1}$ such that there is exactly one vertex between $h_{i}$ and $h_{i+1}$.

Proof. Let us call $y_{1}<\cdots<y_{n}$ the heights of the $n$ vertices in $\Gamma$. Any choice of $h_{i}$ such that $h_{1}<y_{1}<h_{2}<\cdots<h_{n}<y_{n}<h_{n+1}$ satisfies the property.

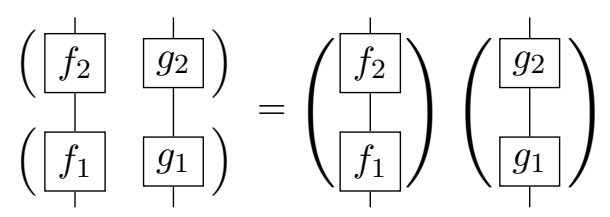

FIgURE 4. The bifunctoriality equation in string diagrams

$$
b \bullet \quad=a_{a \bullet}^{\bullet}=a \bullet b \bullet=\begin{aligned}
& a \bullet \\
& a \bullet
\end{aligned} \quad \begin{aligned}
& a \bullet \\
& b
\end{aligned}
$$

FiguRE 5. The Eckmann-Hilton argument in string diagrams 
1.3. Combinatorial encoding of string diagrams. Our aim is to give complexity results about problems involving string diagrams. We must therefore propose an efficient encoding for them and define the computational model that we use to measure time complexity of our algorithms. We will use the RAM model, used for instance by [HW74], which assumes constant time random access to the working memory and constant time arithmetic operations on integers. This is a widespread model, which closely matches the architecture of today's computers, despite the fact that arithmetic operations on unbounded integers would not be implementable in constant time. We discuss the implications of this feature in Sections 6 and 7 .

Lemma 1.7 provides the basis for a compact encoding of string diagrams. The main idea is that a diagram is cut into slices given by the lemma, and each slice can be described by the following data:

- the number of wires at the top and bottom of the slice (which we will not directly encode as they are redundant with the neighbouring slices);

- the number of input and output wires for the generator in the slice;

- the horizontal position of the generator, described for instance by the number of wires passing to the left of the generator;

- the generator morphism itself, denoted by an identifier taken from the signature. For our purposes this will be omitted to simplify the presentation, as our results are applicable to any signature.

This encoding scheme is essentially identical to that used by the proof assistant Globular [BV16], although the result in this section is new, and is not implied by the existing literature. This encoding scheme serves as a formal combinatorial foundation for our results, although we will build most of our arguments at a more intuitive level with the corresponding graphical diagrams.

We give an example of a diagram, together with its encoding, in Figure 6. Note that in this example diagram, and in the other diagrams in the paper, we use small circles for the vertices, rather than boxes which are sometimes seen.

Definition 1.8. For a natural number $n \in \mathbb{N}$, we define the total order $[n]=\{0, \ldots, n-1\}$.

Definition 1.9. A diagram $D=(D . S, D . N, D . H, D . I, D . O)$ comprises $D . S \in \mathbb{N}$, the number of source edges; D.N $\in \mathbb{N}$, the diagram height; and functions D.H,D.I, D.O : $[D . N] \rightarrow \mathbb{N}$ of vertex horizontal positions, vertex source size and vertex target size respectively.

Given a diagram, we can compute the number of edges that exist at level just above each vertex, by starting with the number of source edges D.S, and then supposing that each vertex $n \in[D . N]$ removes $D . I(n)$ wires and adds $D . O(n)$ wires. We develop that formally as follows.

Definition 1.10. For a diagram $D$, we define $D . \Delta:[D \cdot N] \rightarrow \mathbb{N}$ as $D \cdot \Delta(n)=D . O(n)-$ D.I $(n)$.

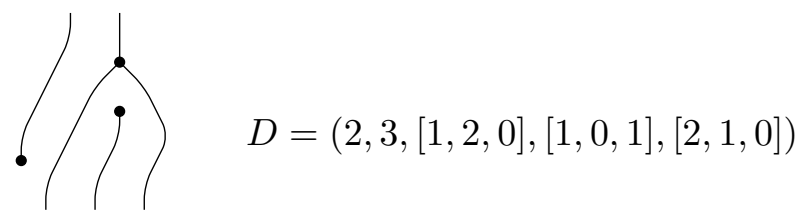

FiguRE 6. Example of a diagram $D$ together with its encoding. 
Definition 1.11 (Wires at each level). For a diagram $D$, we define $D . W:[D \cdot N+1] \rightarrow \mathbb{N}$ as $D . W(0)=D . S$, and for $n \in[D \cdot N]$ as $D \cdot W(n+1)=D \cdot W(n)+D \cdot \Delta(n)$.

Not all diagrams will be geometrically meaningful, and we give validity conditions which check that there are "enough edges" above each vertex to serve as its source edges.

Definition 1.12. A diagram $D$ is valid when for all $n \in[D . N]$, we have $D . W(n) \geq D . H(n)+D . I(n)$.

We now formalize the right and left exchange moves illustrated in Figure 7. All that needs to be checked is that the two vertices with adjacent heights share no common edges.

Definition 1.13. For $n \in[D . N-1]$, a diagram $D$ admits a left exchange move at height $n$ when $D . H(n+1) \geq D \cdot H(n)+D . O(n)$, and admits a right exchange move at height $n$ when $D \cdot H(n) \geq D \cdot H(n+1)+D \cdot I(n+1)$. A right reduction is a series of right exchange moves.

Definition 1.14. For a diagram $D$ which admits a left or right exchange move at height $n \in[D . N-1]$, its left exchange $D_{\mathrm{L}, n}$ or right exchange $D_{\mathrm{R}, n}$, respectively, is defined to be identical to $D$, except at heights $n, n+1$ as follows:

$$
\begin{aligned}
D_{\mathrm{L}, n} \cdot H(n) & =D \cdot H(n+1)-D \cdot \Delta(n) \\
D_{\mathrm{L}, n} \cdot I(n) & =D \cdot I(n+1) \\
D_{\mathrm{L}, n} \cdot O(n) & =D \cdot O(n+1) \\
D_{\mathrm{L}, n} \cdot H(n+1) & =D \cdot H(n) \\
D_{\mathrm{L}, n} \cdot I(n+1) & =D \cdot I(n) \\
D_{\mathrm{L}, n} \cdot O(n+1) & =D \cdot O(n) \\
D_{\mathrm{R}, n} \cdot H(n) & =D \cdot H(n+1) \\
D_{\mathrm{R}, n} \cdot I(n) & =D \cdot I(n+1) \\
D_{\mathrm{R}, n} \cdot O(n) & =D \cdot O(n+1) \\
D_{\mathrm{R}, n} \cdot H(n+1) & =D \cdot H(n)+D \cdot \Delta(n+1) \\
D_{\mathrm{R}, n} \cdot I(n+1) & =D \cdot I(n) \\
D_{\mathrm{R}, n} \cdot O(n+1) & =D \cdot O(n)
\end{aligned}
$$

Lemma 1.15. For a valid diagram $D$ which admits a right (or left) exchange move at height $n$, its right exchange $D_{\mathrm{R}, n}$ (or left exchange $D_{\mathrm{L}, n}$ ) is a valid diagram.

Proof. Let $D$ be a valid diagram which admits a right exchange at height $n$. We prove that $D_{R, n}$ is valid again. The case of left exchanges is symmetrical.

We need to check that for each height $k \in\left[D_{R, n} \cdot N\right]$, we have $D_{R, n} \cdot H(k)+D \cdot I_{R, n}(k) \leq$ $D_{R, n} . W(k)$.

For $k<n$ or $k>n+1, D_{R, n} \cdot H(k)=D \cdot H(k), D_{R, n} \cdot I(k)=D \cdot I(k)$ and $D_{R, n} \cdot W(k)=$ $D . W(k)$ so by validity of $D$ the inequality holds.

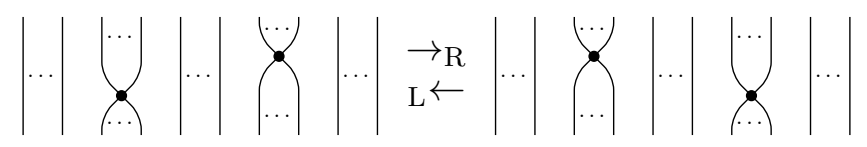

FiguRE 7 . Right and left exchanges as rewrites on diagrams. 
For $k=n$, by definition of $D_{R, n}$ we have $D_{R, n} \cdot H(n)+D_{R, n} \cdot I(n)=D \cdot H(n+1)+$ $D . I(n+1)$. As $D$ admits a right exchange at height $n$, this is bounded by $D \cdot H(n)$, so a fortiori $D . W(n)$.

For $k=n+1, D_{R, n} . H(n+1)+D_{R, n} \cdot I(n+1)=D \cdot H(n)+D \cdot O(n+1)-D \cdot I(n+1)+D \cdot I(n)$. By validity of $D$, this is bounded by $D \cdot W(n)+D \cdot O(n+1)-D \cdot I(n+1)=D_{R, n} \cdot W(n+1)$.

It is a consequence of Theorem 1.4 that right and left exchanges preserve the meaning of the diagram. With respect to our data structure described here, it is clear that the following operations can be performed in constant time, since they involve computing fixed formulae over the natural numbers, and testing a fixed number of inequalities:

- checking whether a left or right exchange is admissible at a given height;

- given an admissible left or right exchange, computing the rewritten diagram in place.

Furthermore, the memory space needed to represent a diagram is linear in the number of vertices. We will use these observations as building blocks for our complexity arguments in the main part of the paper.

As indicated in Figure 7, we write $\rightarrow_{\mathrm{R}}$ and $\rightarrow_{\mathrm{L}}$ for the relations on diagrams given by a single right exchange and left exchange respectively. We illustrate some interesting cases of these exchange moves. In degenerate cases where $u$ and $v$ have no inputs or outputs, it can be possible to apply two right exchanges in sequence to the same pair of vertices:

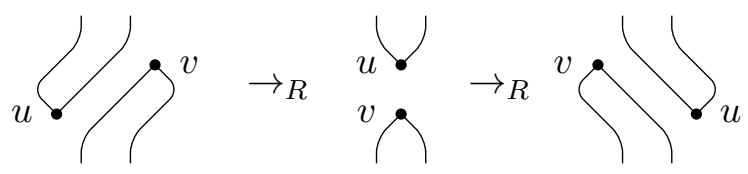

Furthermore, if there are no edges at all, then right exchanges can be applied indefinitely, which corresponds to the Eckmann-Hilton argument shown in Section 1.2.

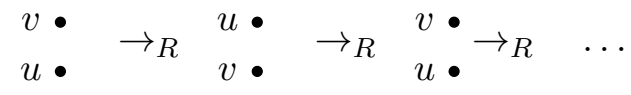

Throughout this article we will use a braid notation to represent right reductions (series of right exchanges), such as in Figure 8 or Figure 9. These braidings represent the trajectory of the vertices as the reduction progresses, seen from the right-hand side of the diagram. Each crossing in the braid diagram corresponds to an exchange of two nodes in the string diagram.

1.4. Converting between representations of morphisms. This combinatorial encoding given in this section can be rendered into an actual diagram. Generating such a representation from our encoding involves computing suitable planar layouts for the vertices and edges respecting all the properties of this class of topological graphs. Various algorithms can be used to this end. In this work we use a simple layout strategy that enforces a constant vertical spacing between diagram levels and a constant horizontal spacing between the wires at each level. ${ }^{4}$ Each level is horizontally centered based on the number of wires that cross

\footnotetext{
${ }^{4}$ It is simple enough to be programmed in ${ }^{A A} \mathrm{~T}_{\mathrm{E}} \mathrm{X}$, so that our string diagrams are generated with this rendering process, directly from their combinatorial encodings.
} 
it. Vertices are horizontally centered between their input and output ports. An example of such a rendered diagram can be found in Figure 6 .

It is also possible to convert a morphism expression into a combinatorial encoding of its string diagram in linear time.

Theorem 1.16. Two morphisms expressions denote the same morphism if and only if the corresponding diagrams are related by a series of exchanges.

Proof. Theorem 1.4 shows that two morphism expressions denote the same morphism if and only if their string diagrams are related by a deformation of recumbent graphs. Therefore we only need to show that string diagrams are related by a deformation if and only if their combinatorial encodings are related by a series of exchanges.

If two diagrams are related by a series of exchanges, they represent the same morphism as exchanges preserve denotation.

Conversely, let $h$ be a deformation between diagrams $\Gamma$ and $\Gamma^{\prime}$ in generic position, with $h(0)=\Gamma$ and $h(1)=\Gamma^{\prime}$. We can assume that $h(t)$ is always in generic position except for a finite number of $t \in(0,1)$ : if it is not the case, translate each vertex $v_{i}$ vertically by $\epsilon_{i}$, uniformly for all $t \in[0,1]$. The $\epsilon_{i}$ can be chosen to make sure no vertices remain at the same height for a non-trivial interval $t \in[u, v]$.

Furthermore, we can make sure that when $h(t)$ is not in generic position, only two vertices in $h(t)$ are at the same height. If this is not the case, the deformation can be modified to satisfy this condition by picking delays $\eta_{i}$ for each vertex $v_{i}$, and delaying the movement of each vertex $v_{i}$ by $\eta_{i}$ over the course of the transformation. Again, the delays can be chosen collectively to ensure that at most two vertices occupy the same height at a given time.

Let $t_{1}<\cdots<t_{k}$ be the instants at which $h(t)$ is not in generic position. For any other $t \in[0,1]$ the combinatorial encoding of $h(t)$ is defined. By connectedness, the combinatorial encoding of $h(t)$ is constant for $t \in\left(t_{i}, t_{i+1}\right)$ so this defines a sequence of diagrams $D_{0}, \ldots, D_{k}$. Since at most one pair of vertices exchange heights around instant $t_{i}, D_{i}$ and $D_{i+1}$ are related by a single exchange move or are equel. This gives the required sequence of exchanges between the source and target diagrams.

Therefore, solving the word problem for free monoidal categories can be done by providing an algorithm to determine if two diagrams can be related by a series of exchanges. We will first show that right reductions form a terminating and confluent rewriting strategy on connected diagrams. Termination will be shown in Section 2 and confluence in Section 4.
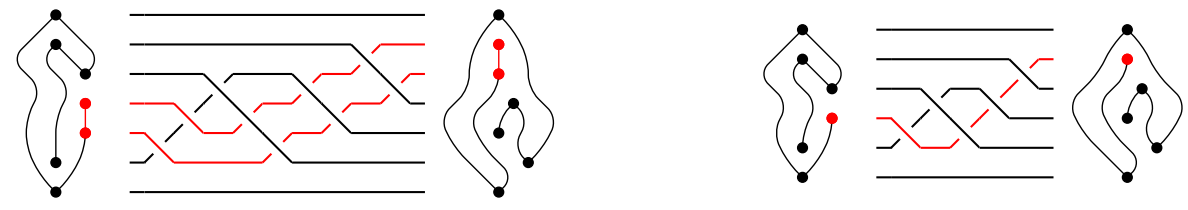

FigURE 8. A collapsible reduction and its collapsed counterpart 


\section{TERmination}

To prove termination of right reductions on connected diagrams, we first introduce the class of linear diagrams, which we will study before tackling the general case. We will see in Lemma 3.5 that they exhibit the longest reductions.

Definition 2.1. A diagram with $n$ vertices is linear if it is connected, acyclic and has only two leaves (vertices connected to only one edge). We identify its vertices with the indices $1, \ldots, n$ such that 1 and $n$ are the leaves, and $k$ is connected to $k-1$ and $k+1$ for all $1<k<n$.

As the name indicates, linear diagrams have a path-like shape (since they are connected and cannot contain any branching). Figure 10 gives an example of a linear diagram. The choice of the start and end of the indexing is arbitrary, as it can be reversed. We therefore assume that linear diagrams come with a chosen order.

Definition 2.2. In a linear diagram with $n$ vertices, $n \geq 2$, the final vertices are the vertices $n-1$ and $n$.

Definition 2.3. In a linear diagram, the final interval is the set of vertices whose height is between the heights of the final vertices, including the final vertices themselves. If the final interval only consists of the final vertices, the diagram is collapsible.

In Figure 10, vertices 1 and 3 are in the final interval, as well as the final vertices themselves, vertices 4 and 5 .

Definition 2.4. A right reduction is collapsible when its source and target are collapsible, and any exchange between a non-final vertex $v$ and a final vertex $f_{1}$ is immediately followed or preceded by an exchange between $v$ and the other final vertex $f_{2}$. In other words, all non-collapsible steps of the reduction are isolated.

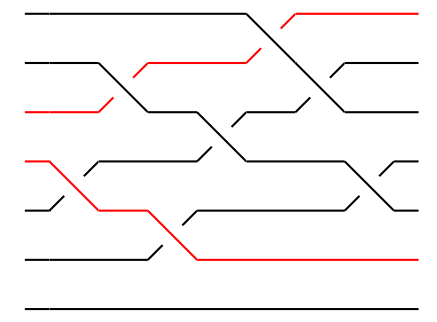

(A) A funnel with collapsible source

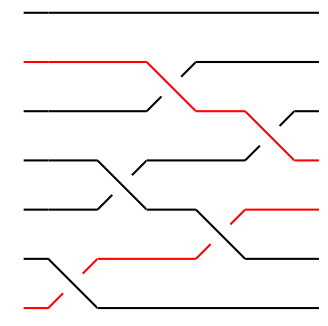

(B) A funnel with collapsible target

Figure 9. Example of funnels

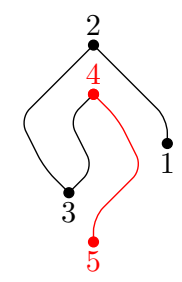

FiguRE 10. Example of a linear diagram, with final vertices in red 
We call these reductions collapsible because as the final vertices move synchronously, they can be merged together: this defines a reduction on a shorter linear diagram. Figure 8 shows an example of a collapsible reduction with the final vertices in red, and the corresponding collapsed reduction on the shorter diagram.

Definition 2.5. Given a collapsible reduction on a linear diagram $l$ of size $n$, the corresponding collapsed reduction is obtained by erasing vertex number $n$ in $l$.

Definition 2.6. A right reduction of string diagrams $r: A \rightarrow_{\mathrm{R}}^{*} B$ is called a funnel when:

- each non-final vertex is exchanged at most once with a final vertex.

- if an exchange involves non-final vertices $u$ and $v$, then both $u$ and $v$ are exchanged with a final vertex in the course of the rewrite, and these two final vertices are different.

We are especially interested in the cases where the source or target of the funnel is collapsible, as in Figure 9. The name funnel comes from the shape of these reductions when depicted as braids: these are reductions where the final vertices converge or diverge from each other.

The following lemmas will establish various properties of funnels that we will need for the decomposition of Lemma 2.12.

Lemma 2.7. Let $r: A \rightarrow_{\mathrm{R}}^{*} B$ be a funnel with $A$ collapsible and $e: B \rightarrow_{\mathrm{R}} C$ be a right exchange of two non-final vertices $u$ and $v$ that are not touched by $r$. Then the reduction $r ; e: A \rightarrow_{\mathrm{R}}^{*} B \rightarrow_{\mathrm{R}} C$ can be rearranged as $e^{\prime} ; r^{\prime}: A \rightarrow_{\mathrm{R}} B^{\prime} \rightarrow_{\mathrm{R}}^{*} C$, where $e^{\prime}$ exchanges $u$ and $v$ in $A$, and $r^{\prime}$ is a funnel.

Proof. As $u$ and $v$ are not touched by $r$, the two reductions commute directly.

Lemma 2.8. Let $r: A \rightarrow_{\mathrm{R}}^{*} B$ be a funnel reduction where $A$ or $B$ is collapsible. Then, the trajectory of all non-final vertices is monotone in $r$.

Proof. Let us assume by symmetry that the source $A$ of the reduction is collapsible. Consider an exchange of non-final vertices $u$ and $v$ in $r$. By definition, $u$ and $v$ are exchanged with two different final vertices over the course of $r$. Because $A$ is collapsible, this means that both $u$ and $v$ have entered the final interval earlier in the reduction, by being exchanged with the bottom and top final vertices (respectively). Figure 11 shows the general position of such an exchange.

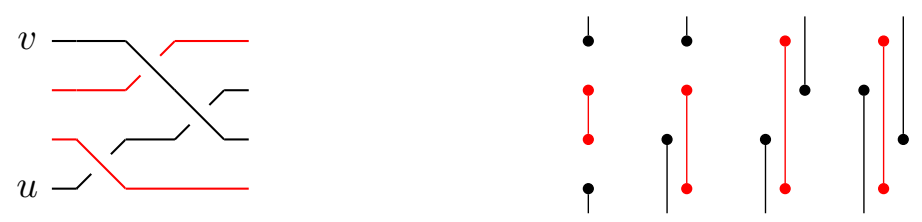

(A) The general position of an exchange (B) Relative horizontal positions of nodes of final vertices in $r$

in $r$

FIGURE 11. Horizontal position of non-final nodes in a funnel

As all the exchanges involved are right exchanges, $u$ and $v$ are on different sides of the final edge when they are exchanged: $u$ is on the left and $v$ is on the right of the final edge. This means that $u$ necessarily goes up and $v$ goes down. As this applies to all exchanges of non-final vertices, this means that the trajectory of both vertices is monotone. 
Definition 2.9. An interval right exchange $i: A \rightarrow_{\mathrm{R}}^{*} B$ is a right reduction swapping a vertex $x$ and a set of consecutive vertices $v_{1}, \ldots, v_{k}$ which is adjacent to $x$ in $A$ and $B$. The vertex $x$ is exchanged first with $v_{1}$, then $v_{2}$, up to $v_{k}$.

An interval right exchange looks like this:

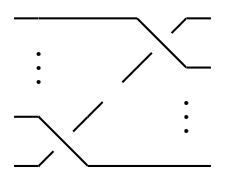

Lemma 2.10. Let $r: A \rightarrow_{\mathrm{R}}^{*} B$ be a funnel reduction with $A$ collapsible and $e: B \rightarrow_{\mathrm{R}} C$ be an exchange of a non-final vertex $v$ with a final vertex $f_{2}$, such that $v$ is exchanged with the other final vertex $f_{1}$ in $r$. This gives a reduction path $r ; e: A \rightarrow_{\mathrm{R}}^{*} B \rightarrow_{\mathrm{R}} C$. A reduction of the same length can be obtained: $i ; r^{\prime}: A \rightarrow_{\mathrm{R}}^{*} D \rightarrow_{\mathrm{R}}^{*} C$ where $r^{\prime}$ is a funnel reduction and $i$ exchanges $v$ with the final interval in $A$.

Proof. By symmetry let us assume that $f_{1}$ is the highest final vertex, and $f_{2}$ is the lowest. Somewhere in $r, v$ enters the final interval by being exchanged with $f_{1}$. By Lemma 2.8, the trajectory of $v$ in $r$ is monotone. In fact, because $v$ ends up being adjacent to $f_{2}$ in $B, v$ is exchanged exactly once with each non-final vertex that is exchanged with $f_{2}$ over the course of $r$.

Exchanges that do not involve $v$ can be divided in two blocks: the ones that are on the right of the trajectory of $v$, and the ones that are on the left. The block on the right commutes with $e$ because the vertices they exchange are disjoint, so we can permute the two.

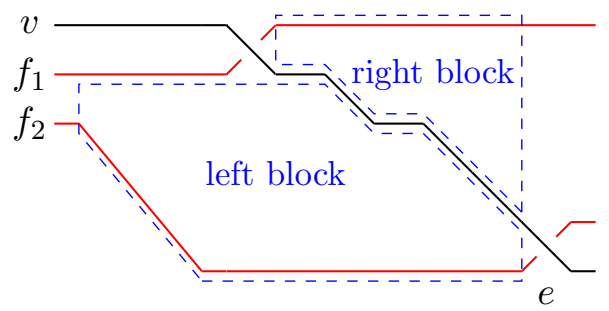

We now need to pull the block on the left through the exchanges involving $v$. Notice that $v$ is the first vertex to be exchanged with $f_{1}$ over the course of $r$. This is because all other such vertices cannot be exchanged with $v$ in $f$ and $v$ is adjacent to $f_{2}$ in $B$. Thus, the block on the left does not contain any exchange involving $f_{1}$ : it only contains exchanges involving non-final vertices or $f_{2}$. By successive application of the Reidemeister type III move (which pulls one exchange through two other exchanges), we can therefore pull the left block through the trajectory of $v$.

Lemma 2.11. Let $r: A \rightarrow_{\mathrm{R}}^{*} B$ be a funnel reduction with $A$ collapsible, followed by an exchange $e: B \rightarrow_{\mathrm{R}} B^{\prime}$ of two non-final vertices $u, v$ such that both vertices are exchanged with the same final vertex $f$ in $r$. Then, the sequence $r$; e can be rewritten as $e^{\prime} ; r^{\prime}: A \rightarrow_{\mathrm{R}} A^{\prime} \rightarrow_{\mathrm{R}}^{*}$ $B^{\prime}$ where $e^{\prime}$ exchanges $u$ and $v$ in $A$, and $r^{\prime}$ is a funnel.

Proof. We show that $e$ can be pulled through all exchanges involving $u$ or $v$ in $r$. By symmetry, we will assume that the final vertex $f$ exchanged with $u$ and $v$ is the lowest one, and that $u$ is the vertex below $v$ in $B$. 
By induction, consider the last exchange in $r$ that involves one of $u$ or $v$ and another vertex $x$. Because the trajectories of $u$ and $v$ always go up by Lemma 2.8, the trajectory of $x$ goes down. As $u$ and $v$ are adjacent in $B$, this last exchange must be between $u$ and $x$, and $x$ must have been exchanged previously with $v$. Moreover, this previous exchange is necessarily the last one involving $v$ (otherwise any later exchange with $y$ would require a later exchange between $y$ and $u$ ). Therefore, $e$ can be pulled through the last exchanges involving $u$ and $v$.
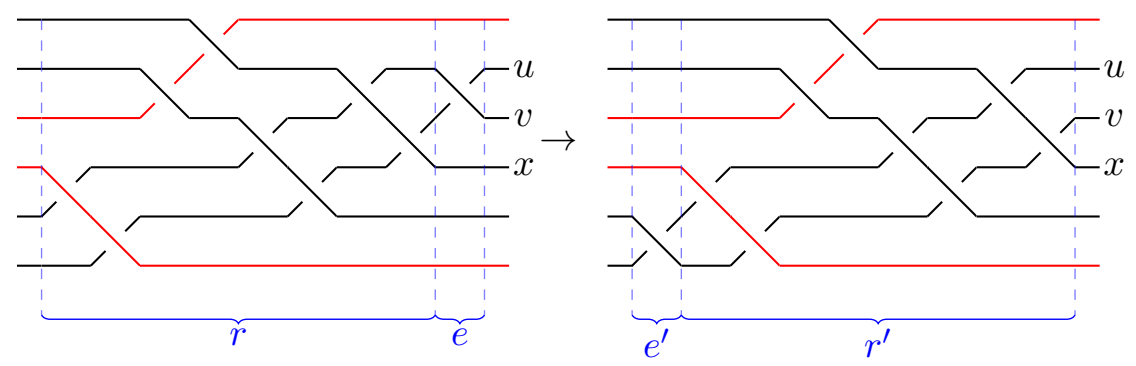

We perform these pull-through moves inductively, which eventually moves $e^{\prime}$ at the beginning of the reduction. The subsequent exchange the same nodes as $r$ in the same order, so they form a funnel.

Finally, the following lemma decomposes reductions on linear diagrams into two parts: a collapsible part and a funnel part. This decomposition is illustrated by Figure 14. As a collapsible reduction can be seen as a reduction on a shorter linear diagram, this will let us work inductively on the size of the linear string diagram.

Lemma 2.12. Let $r: A \rightarrow_{\mathrm{R}}^{*} B$ be a reduction with $A$ collapsible. Then $r$ can be rearranged and decomposed as

$$
c ; f: A \rightarrow_{\mathrm{R}}^{*} X \rightarrow_{\mathrm{R}}^{*} B
$$

with c collapsible and $f$ a funnel.

Proof. We construct the decomposition into collapsible and funnel parts by induction on the length of the rewrite $r$. For length 0 , the result is clear. For length 1, there are two cases: if the exchange touches a final vertex, then it goes in the funnel part of the decomposition, otherwise it forms the collapsible part.

Assume we have a rewrite of length $k+1$. Use the induction hypothesis to decompose the first $k$ exchanges:

$$
c ; f ; z: A \rightarrow_{\mathrm{R}}^{*} X \rightarrow_{\mathrm{R}}^{*} B^{\prime} \rightarrow_{\mathrm{R}} B
$$

with $c$ collapsible and $f$ a funnel.

If $f ; z$ is also a funnel, then this gives us the required decomposition. Otherwise, this funnelity can fail for multiple reasons.

First, it can be that $z$ exchanges a final vertex $v$ with a non-final vertex $w$ that is already exchanged with a final vertex in $f$. In this case, by Lemma 2.10 , we can rearrange $f ; z$ into $i$; $f^{\prime}$ where $f^{\prime}$ is a funnel and $i$ exchanges $v$ with the final interval. As the domain of $i$ is collapsible, $i$ is collapsible itself so we have the required decomposition.

Second, it can be that $z$ exchanges two non-final vertices that are not exchanged with any final vertex in $f$. In this case, by Lemma $2.7, z$ commutes with $f$ : we obtain $c ; z ; f: A \rightarrow_{\mathrm{R}}^{*} X \rightarrow_{\mathrm{R}} X^{\prime} \rightarrow_{\mathrm{R}}^{*} B$, and $c ; z$ is collapsible so we have the required decomposition. 
It cannot be the case that only one of the two non-final vertices $z$ exchanges has been previously exchanged with a final vertex in $f$. This is because the heights of all vertices which have been exchanged with a final vertex lie in the final interval, and all other non-final vertices are outside the final interval.

Third, it can be that $z$ exchanges two non-final vertices that are both exchanged in $f$ with a final vertex. In this case, as we have assumed that $f ; z$ is not final, it must be the vertices were exchanged with the same final vertex. We can therefore apply Lemma 2.11 and rearrange the rewrite into $e^{\prime} ; f^{\prime}$ with $e^{\prime}$ exchanging the same non-final vertices as $z$ and $f^{\prime}$ funnel. As $e^{\prime}$ is collapsible, this gives the required decomposition.

Finally, it cannot be the case that $z$ exchanges the two final vertices, as final vertices can never be exchanged together since they are connected by an edge.

Lemma 2.13. Let $r: A \rightarrow_{\mathrm{R}}^{*} B$ be a right reduction on a linear diagram. Then $r$ can be extended on some side such that its domain or codomain is collapsible.

Proof. Our strategy to extend $r$ depends on the topology of the final vertices. We know that vertex $n$ is connected solely to $n-1$ and that $n-1$ is connected to both $n-2$ and $n$. Here are the possible ways these connections can happen:

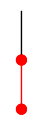

(A)

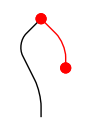

(B)

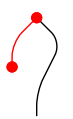

(c)

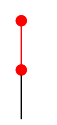

(D)

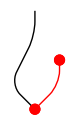

(E)

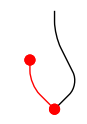

(F)

The orientation of the edges involved is preserved by the reductions so the same situation is observed in both $A$ and $B$.

Consider situation (a). If the terminal layout $B$ is not collapsible, non-final nodes are present between $n$ and $n-1$. Some of them are on the left side of the edge connecting the final vertices and the others are on the right-hand side. Any two such nodes which are not on the same side of the final edge can be exchanged, so by appending a right reduction to $r$ we can ensure that all the ones on the left are just below $n-1$, and all the ones on the right are just above $n$. Then, by adding further right exchanges, we can move these non-final nodes outside the final interval, leading to a collapsible configuration. This is illustrated in Figure 13a. In the situation illustrated in Figure 13b, we choose instead to prepend right exchanges before $r$ : this is necessary to expell vertices nested inside the cap outside the final interval. The other cases are similar: in each of them, we can either prepend or append right exchanges to obtain a collapsible configuration.

We can now show termination of right reductions. A finer analysis of the bound obtained on the length of reductions is presented in Section 3.

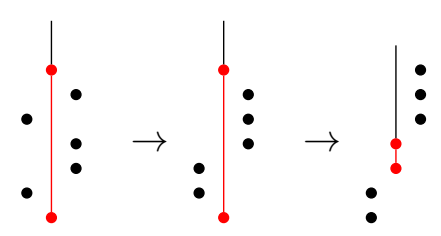

(A) Appending right exchanges

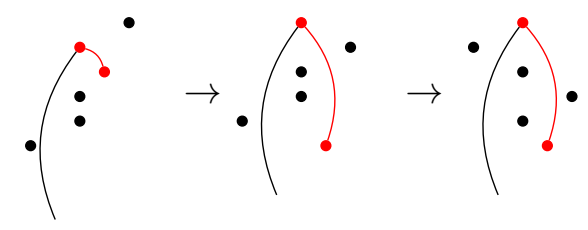

(в) Prepending right exchanges

Figure 13. Extending a reduction so that one end is collapsed 
Theorem 2.14. Right reductions are terminating on connected diagrams.

Proof. We first show termination for linear diagrams. Notice that the length of a funnel reduction on a linear diagram of size $n$ is bounded by $F(n)=O\left(n^{2}\right)$. This is because exchanges involving final vertices happen at most $O(n)$ times and exchanges involving only non-final vertices happen at most once per pair of non-final vertices by Lemma 2.8 .

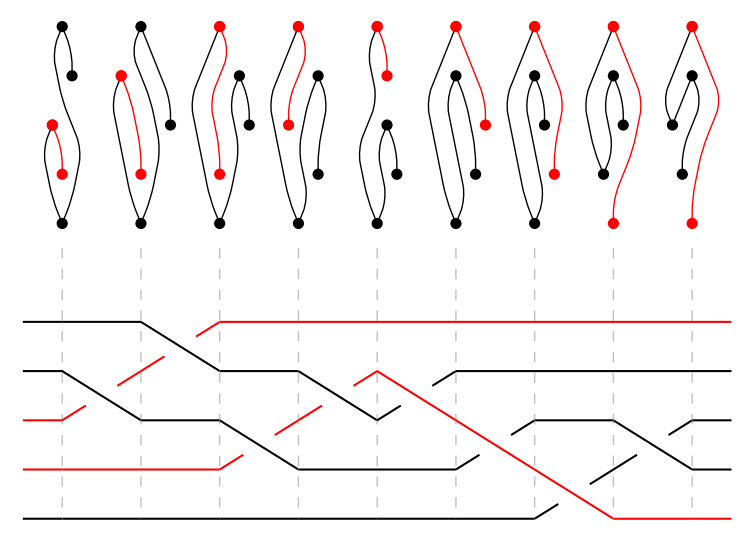

(A) Reducing a diagram to its normal form

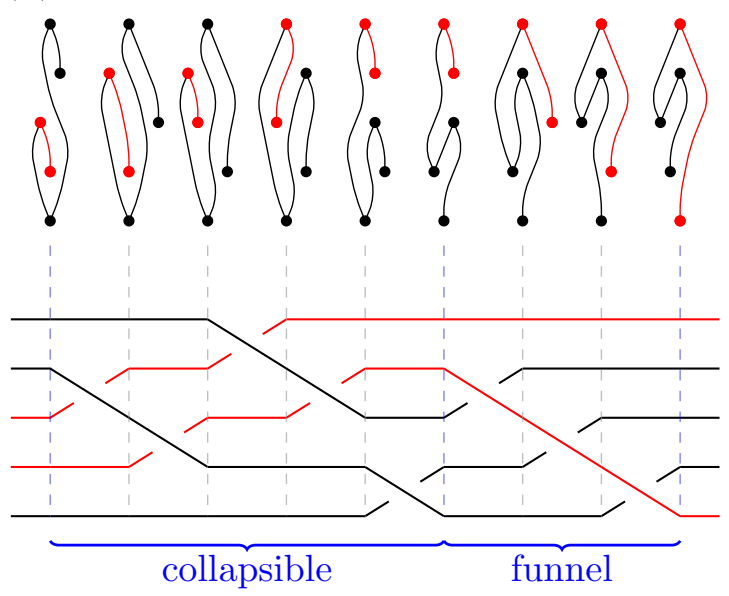

(в) Decomposition from Lemma 2.12

FiguRE 14. Decomposition into collapsible and funnel reductions

We can now define a bound $B(n)$ on the length of right reductions on linear diagrams of size $n$, by induction on $n$. Consider such a reduction $r$. By Lemma 2.13, we can assume that one end of $r$ is collapsible (by making $r$ potentially longer). By Lemma 2.12, we can decompose $r$ into a funnel part $f$ and a collapsible part $c$. The collapsible part $c$ gives rise to a collapsed reduction $c^{\prime}$, whose length is bounded by $B(n-1)$ by induction. Because an exchange involving the last vertex in the shorter diagram corresponds to two exchanges in the longer diagram, we obtain $|c| \leq 2 B(n-1)$. By the observation above, $|f| \leq F(n)$. Hence, $|r| \leq 2 B(n-1)+F(n)=: B(n)$. This shows termination of right reductions on linear diagrams. 
We now move to the general case of connected diagrams. Assume by contradiction that there is an infinite reduction on a connected diagram. By the pigeonhole principle, there is a pair of vertices that are exchanged infinitely often. Consider a simple path between these two vertices and erase all vertices not visited by this path. The infinite reduction on the connected diagram induces an infinite reduction on the linear diagram, which contradicts termination on linear diagrams.

Some diagrams are not connected as graphs but all their vertices are connected to a boundary. Theorem 2.14 can be extended to these cases.

Definition 2.15. A diagram $D$ is boundary-connected if it is connected or all vertices in $D$ are connected to one of the two boundaries of the diagram.

Figure 15a shows a diagram that is not connected (it has three connected components) but which is boundary-connected, since each component contains an open wire. Each vertex is therefore connnected to either the top or bottom boundary of the diagram via these open wires.

Corollary 2.16. Right reductions on boundary-connected diagrams are terminating.

Proof. Let $D$ be boundary-connected. Consider the diagram $D^{\prime}$ obtained from $D$ by adding two vertices $b, t$ at the bottom and top boundaries, and adding two edges from $b$ to $t$ on each side of the diagram, as in Figure 15. Every edge connected to the boundary in $D$ is connected to one of $b, t$ in $D^{\prime}$, so $D^{\prime}$ is connected. Any right reduction on $D$ induces a reduction of the same length on $D^{\prime}$, therefore right reductions on $D$ terminate.

\section{UPPER BOUND ON REDUCTION LENGTH}

Beyond termination, we can use the same proof techniques to derive an asymptotic bound on reduction length. We first introduce a parametric cost on exchanges of linear diagrams:

Definition 3.1. Given a reduction $r$ on a linear diagram of size $n$ and an integer $w$, the cost of $r$ at weight $w$ is $X+w Y$, where $X$ is the number of exchanges not involving vertex number $n$ in $r$ and $Y$ is the number of exchanges involving vertex $n$ in $r$.

Lemma 3.2. The maximum cost at weight $w$ of a funnel with a collapsible end is $f(n, w)=$ $O\left(n^{2}+w n\right)$, where $n$ is the length of the linear diagram.

Proof. A funnel contains two types of exchanges. Those with final vertices account for at most $n-2$ exchanges, because there is at most one for each non-final vertex. The ones with only non-final vertices are bounded by $O\left(n^{2}\right)$ as any pair of non-final vertices is exchanged at most once by Lemma 2.8. The bound follows from the definition of the cost.

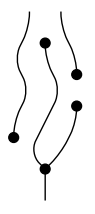

(A) The original diagram $D$

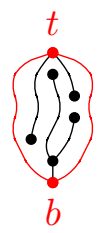

(B) The transformed diagram $D^{\prime}$

FiguRE 15. Adding nodes on the boundaries to make a diagram connected 
Theorem 3.3. The maximum cost of a right reduction on a linear diagram is $O\left(n^{3}+w \cdot n^{2}\right)$, where $n$ is the size of the diagram.

Proof. Let $g(n, w)=\sum_{k=1}^{n} f(k, w+n-k)$. We show that $g(n, w)$ bounds the cost of any right reduction on a linear diagram of size $n$. By Lemma 3.2, the desired bound will follow. We work by induction on $n$. For $n \leq 1$, no right exchanges can be performed, so the bound holds. Consider a reduction $r: A \rightarrow_{\mathrm{R}}^{*} B$ on a linear diagram of size $n$. By Lemma 2.13, we can assume that $A$ or $B$ is collapsible (up to an extension which increases the cost of $r$ ). By Lemma 2.12, we can rearrange the exchanges in $r$ to obtain a funnel and a collapsible reduction. By definition, the cost of the funnel part is bounded by $f(n, w)$. For the collapsible part, consider the reduction induced by merging the final vertices together: this gives a reduction on a diagram of size $n-1$. Each exchange involving the last vertex in this induced reduction corresponds to an exchange of both final vertices in the original reduction, which has cost $w+1$. Therefore, by induction, the cost of the collapsible part is bounded by $g(n-1, w+1)$. We therefore obtain the bound $g(n-1, w+1)+f(n, w)=g(n, w)$ on the cost of $r$ at weight $w$.

Theorem 3.4. The maximum length of a reduction on a diagram of size $n$ vertices is $O\left(n^{3}\right)$.

Proof. By the same argument as Corollary 2.16 we can assume that the diagram is connected. Consider a connected string diagram $D$ with $v$ vertices. Pick a spanning tree on $D$ and let $D^{\prime}$ be the string diagram obtained from $D$ by removing all edges which are not in the spanning tree. Any reduction on $D$ induces a reduction of the same length on $D^{\prime}$, so it is enough to bound the length of reductions on $D^{\prime}$.

Pick an arbitrary vertex of $D^{\prime}$ as root for the tree and consider a depth-first search of $D^{\prime}$ from that root. This defines an envelope on the tree, which can be seen as a linear diagram $L$ if we duplicate the nodes every time they are visited (see Figure 16). The length of this diagram is linear in the number of edges in $D^{\prime}$, which is linear in the number of vertices in $D$ (since $D^{\prime}$ is a tree, it has one less edge than vertices, and its vertices are those of $D$ ).

Any right reduction on $D^{\prime}$ translates to a right reduction on $L$, where exchanging vertices $x$ and $y$ corresponds to exchanging all the copies of $x$ and $y$ in the same way. The reduction on $L$ is therefore at least as long as the reduction on $D^{\prime}$. By Theorem 3.3 and because the number of vertices in $L$ is linear in $v$, the reduction on $L$ has length $O\left(v^{3}\right)$. This bound also applies the original reduction on $D^{\prime}$ and hence on $D$.

This asymptotic bound on reduction length is attained by a class of spiral-shaped diagrams:

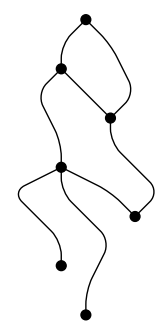

(A) A connected diagram $D$

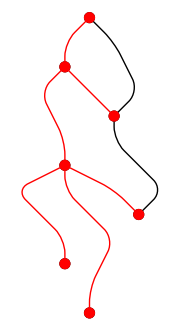

(B) A spanning tree $D^{\prime}$ on $D$ (c) A linear diagram $L$ obtained from $D^{\prime}$

FiguRE 16. Transforming a connected diagram to a linear diagram 


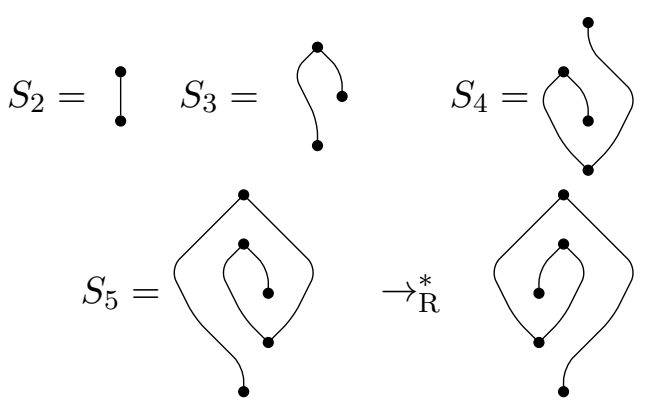

Lemma 3.5. For all $n$, the diagram $S_{n}$ right reduces to its normal form in $\left(\begin{array}{l}n \\ 3\end{array}\right)$ steps.

Proof. A reduction of $S_{n}$ to its normal form starts with $n-2$ exchanges of one end with the rest, followed by the reduction for $S_{n-1}$ where the end weighs one more vertex. Therefore, the cost of a right reduction of $S_{n}$ to its normal form is $s(n, w)=w(n-2)+s(n-1, w+1)$. We also have $s(2, w)=0$ for all $w$. From this we obtain

$$
s(n, w)=\frac{(n-1)(n-2)(n-3+3 w)}{6}
$$

which gives $\left(\begin{array}{l}n \\ 3\end{array}\right)$ for $w=1$.

\section{Confluence}

Lemma 4.1. The right reduction relation is locally confluent.

Proof. Let $F, G, H$ be diagrams with $G_{R} \leftarrow F \rightarrow_{R} H$. If the two pairs of nodes exchanged in the two branches are disjoint, then the exchanges commute and we can close the diagram in one step: we have $H \rightarrow_{\mathrm{R}} K$ and $G \rightarrow_{\mathrm{R}} K$. Otherwise, the rewriting patterns overlap. There are nodes $u, v$ and $w$ in $F$, such that $u$ and $v$ are adjacent and are exchanged to obtain $G$, and $v$ and $w$ are adjacent and are exchanged to obtain $H$. The situation looks like this:

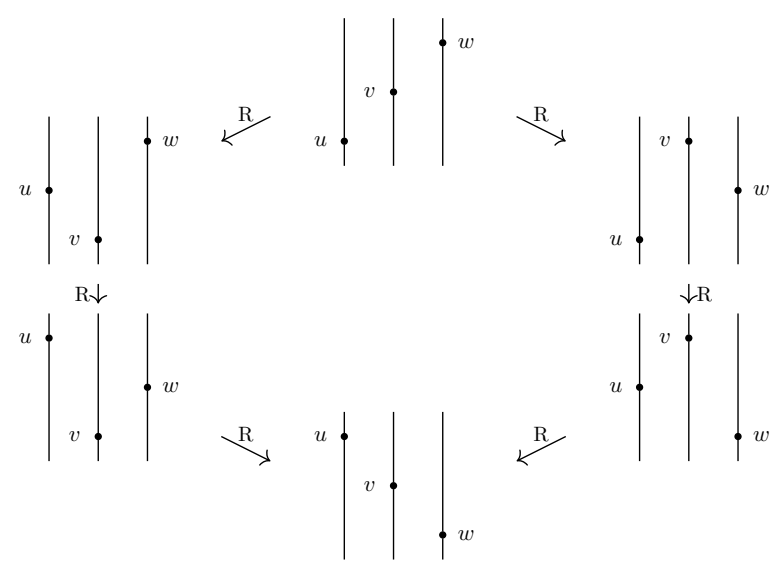

As $u$ and $v$ can be exchanged in $F$, there is no edge from the output of $v$ to the input of $u$, and any edge going from the output of $w$ to the input of $u$ has to pass to the left of $v$. As $v$ and $w$ can be exchanged in $F$, there is no edge from the output of $w$ to the input of $v$, and any edge going from the output of $w$ to the input of $u$ has to pass to the right of $v$, which is 
impossible by the previous observation, so there is no edge from $w$ to $u$. Therefore, $w$ and $u$ can be exchanged both in $G$ and $H$. In the resulting diagrams, we can then exchange $(v, w)$ and $(u, v)$ respectively, which closes the diagram. Note that the braids representation of both sides of the diagram correspond to the Reidemeister type 3 move.

Theorem 4.2. Right reductions are confluent and therefore define normal forms for diagrams under the equivalence relation induced by exchanges.

Proof. By Theorem 3.4 the reduction is terminating and by Lemma 4.1 it is locally confluent, so by Newman's lemma right reductions are confluent. Therefore, the right normal form for a given diagram can be obtained by applying any legal right exchanges until a normal form is reached.

\section{COMPuting NORMAL FORMS}

It follows from Theorem 4.2 that applying the right reduction rewrite strategy allows us to find normal forms in $O\left(v^{4}\right)$ time, where $v$ is the number of vertices: we perform $O\left(v^{3}\right)$ exchanges, each of which can be found and performed in $O(v)$ time. In this section we show that this complexity can be improved, giving a procedure which constructs the normal form directly in $O(v e)$ time, where $e$ is the number of edges.

Let $D$ be a connected diagram in right normal form and $v \in D$ be a vertex. We analyze how a new vertex $l$ can be added to $D$ by connecting it to $v$ only, such that $l$ becomes a leaf in the new diagram. First, we need to choose whether to connect $l$ to the domain or codomain of $v$. Assume for instance that we connect it to the domain of $v$. If $v$ has $k$ edges in its domain before the addition, there are $k+1$ possible positions for the new edge between $l$ and $v$. Assume that such a position is chosen. The height of the vertex $l$ in the new diagram must also be chosen, as shown in Figure 17. The following lemma shows that there is only one such choice such that the new diagram is in right normal form.

Lemma 5.1. Let $D^{\prime}$ be a diagram obtained from $D$ by adding a leaf $l$ connected to a vertex $v \in D$, at a determined side (domain or codomain) and position between existing edges on that side. There is a unique vertical position of $l$ such that $D^{\prime}$ is in right normal form. Furthermore the horizontal position of $l$ at this height is determined by the position between the existing edges of $v$ that we grow it from.

Proof. Let us first show that there is a vertical position for $l$ such that $D^{\prime}$ is in right normal form. First, pick an initial vertical position for $l$, such as the position immediately above or below $v$ (depending on the orientation of the connection between $v$ and $l$ ). Then, normalize
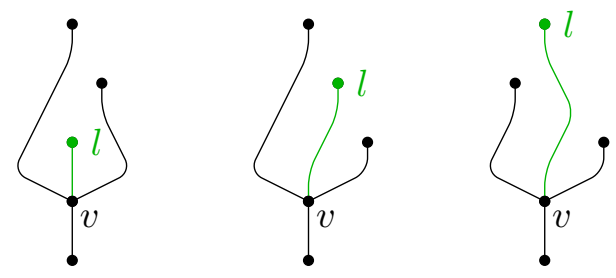

Figure 17. Possible vertical positions to grow a leaf $l$ on $v$. Only the central diagram is in right normal form. 
by applying right exchanges. All the right exchanges involve $l$ : otherwise, by contradiction, consider the first exchange not involving $l$. Removing $l$ from its domain gives us $D$ again (because the relative positions of vertices in $D$ has not changed), and the exchange still applies to this diagram, which contradicts normality of $D$. This shows the existence of the vertical position and uniqueness follows from confluence.

This observation already gives us a way to construct the right normal form of any acyclic connected diagram. For any tree, we can remove one leaf, compute the right normal form of the remaining tree recursively, and add the leaf at the height given by the lemma. However, this does not let us normalize cycles yet.

Definition 5.2. A simple face in a string diagram is a simple edge loop whose inner region does not contain any other vertex or edge. (An edge loop is simple when no edge appears twice in the loop, and each vertex is visited at most once in the loop.)

Definition 5.3. Let $p$ be an oriented path in a diagram. For each vertex $v$ visited by $p$, we define the winding number of $v$ as follows:

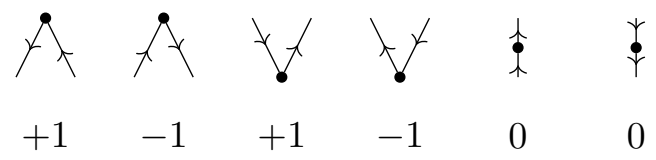

Definition 5.4. Given a simple face in a diagram $D$ and an edge $e$ in the face, the mountain range starting on $e$ is the sequence of partial sums of winding numbers when visiting the face in direct rotation, starting from $e$.

Figure 18 gives an example of a mountain range for an edge in a simple face. Because a cycle forms a closed loop in the plane, the winding numbers of its vertices sums up to two when visited in direct rotation. This means that a mountain range always stops two levels higher than it started.

Definition 5.5. An edge in a simple face is eliminable if the mountain range starting from it never reaches 0 after the first step.

For instance, the edge above is eliminable, but its predecessor is not because the montain range starts with a valley that goes at level -1 and then 0 .

Lemma 5.6. In any simple face there are exactly two eliminable edges.

Proof. Pick an edge in the face and draw the mountain range for it. Let $m$ be the minimum level it reaches after the first step. As the mountain range starts at 0 and ends at $2, m \leq 1$.

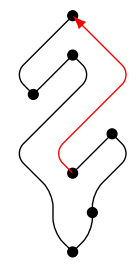

(A) An edge in a face

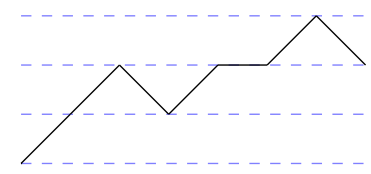

(B) The montain range for this edge

Figure 18. Example of a chosen edge in a face and its mountain range 
Consider the last edge to reach $m$, we will denote it by $e_{1}$. The mountain range on the right of $e_{1}$ never goes below $m+1$ by definition. When drawing the mountain range for $e_{1}$, the left part of the range is shifted upwards by 2 , so this part never goes below $2-m \geq 1$ when drawn as part of the mountain range for $e_{1}$. So $e_{1}$ is eliminable. Similarly, consider the last edge $e_{2}$ to reach 1 in the mountain range starting from $e_{1}$ : it is also eliminable for the same reason. These are the only two edges which satisfy the criterion.

Lemma 5.7. Let $D$ be a connected diagram in right normal form and e be an eliminable edge in a simple face of $D$. Then the diagram $D^{\prime}$ obtained from $D$ by removing e is in right normal form.

Proof. Consider such an edge. We first analyze what it means to be eliminable in geometrical terms. Let us call $u$ the starting point of $e$ and $v$ its end point. We know that $e$ is immediately followed by a left turn (winding number +1 ) at $v$. The next vertex where a rotation happens $w$ also has winding number +1 (otherwise the number of rotations from $e$ to the edge after $w$ would be null). By symmetry let us assume that $e$ points upwards when travelling in the direct orientation on the face.

There are three sorts of right exchanges that could potentially be enabled by removing $e$.

Exchanging $u$ and $v$. The first one would be exchanging the endpoints of $e$ together, but this is impossible because of the left turn on $v$ which imposes a horizontal ordering: no such right exchange can be made.

Exchanging $u$ or $v$ with another vertex $x$. The second one would be exchanging one of the endpoints of $e$ with another vertex. This other vertex must be in the interval between the endpoints (otherwise the exchange was already possible before). That is not possible for $v$ because of the left turn on this vertex. For $u$, this would require having another vertex $x$ immediately to the left of $e$ with no edge linked from below. We will see in a later paragraph that this is not possible.

Exchanging two vertices $x, y$ distinct from $u$ and $v$. Finally, the third case consists in exchanging two nodes $x$ and $y$ between $u$ and $v, x$ immediately to the left of $e$ with no edge linked from below, and $y$ immediately to the right of $e$ with no edge from above. We will show that no such $x$ exists.

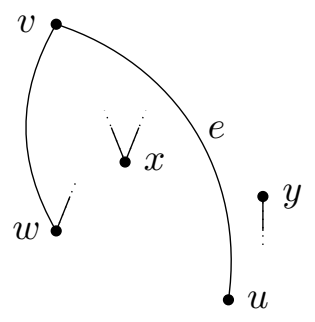


Ruling out the existence of $x$. Because $e$ is the right boundary of the face, such an $x$ must be a part of the boundary of the face. As part of this cycle, it has two edges coming from above. Browsing the cycle in the direct orientation can visit $x$ in two directions: from left to right or from right to left.

If $x$ is visited from left to right, this contradicts the fact that $x$ is immediately to the left of $e$, because the interior of the face is contained between the two edges linked to $x$.

If $x$ is visited from right to left, consider the path from $w$ to $x$. It starts upwards and ends downwards, so it has odd winding number. As $x$ itself is a right turn, this number cannot be negative: otherwise, travelling from $e$ to the edge following $x$ would have null or negative winding number, contradicting the assumption that $e$ is eliminable. So, the path from $w$ to $x$ has positive winding number, and therefore one edge in this path is located between $x$ and $e$, which contradicts the fact that $x$ is immediately to the left of $e$.

Theorem 5.8. The right normal form of a boundary-connected string diagram in free monoidal categories can be decided in time $O(v e)$ where $v$ is the number of vertices and $e$ is the number of edges.

Proof. Again we can restrict our attention to the case of connected diagrams thanks to the reduction of Figure 15. We construct the right normal form of any connected string diagram by induction on the number of edges. The initial case (no edge) is clear.

Given a diagram $D$, there are two cases. We can check in $O(v)$ if $D$ has a leaf, in which case we remove this leaf and obtain a diagram $D^{\prime}$ with one less edge that we can inductively normalize. Then, by Lemma 5.1, we can deduce the right normal form for $D$, by inserting back the leaf at the unique spot which makes the diagram normalized. Such a spot can be found in $O(v)$ by applying right exchanges on the leaf as long as they are admissible. If $D$ does not have any leaf, then it has a simple face. In that case, by Lemma 5.6, there are two eliminable edges in this face. These can be identified in $O(v)$ thanks to the characterization via mountain ranges. We can remove one of them, obtaining diagram $D^{\prime \prime}$, and inductively normalize $D^{\prime \prime}$. By uniqueness of the normal form for $D^{\prime \prime}$ and by Lemma 5.7 , the normal form for $D^{\prime \prime}$ can be obtained by normalizing $D$ and then removing the edge. So the normal form for $D$ can be reconstructed from the normal form for $D^{\prime \prime}$ by adding the edge back. This can also be computed in $O(v)$. We therefore obtain a normalizing algorithm with $e$ induction steps, each of which takes $O(v)$ time, so the overall complexity is $O(v e)$.

\section{Extension to Disconnected Diagrams}

The connectivity requirement is crucial to obtain termination of right reductions and therefore the right normal forms on which we relied on for our results. In this section, we extend our results to arbitrary diagrams. Our approach is to define a complete invariant for the exchange rule.

In general, a digram can contain multiple connected components. Because we are dealing here with non-symmetric monoidal categories, the way these components nest into each other's faces matters as this tree structure is preserved by exchanges. In Appendix A, we define notions of faces, components and enclosure relations between them from the combinatorial representation of diagrams. Each diagram is then represented by a structural tree as in Figure 19, where face nodes have an unordered set of component children and component nodes have an ordered list of face children. We show in Appendix A.1 that such a tree is a complete invariant for exchanges. 
6.1. Word problem. We show how to compute the structural tree of a diagram, and therefore solve the word problem in the general case. Algorithm 6.1 scans the diagram in one pass and computes simultaneously the components and faces of the diagram, as well as the inclusion relation between them. Appendix A defines components and faces as equivalence classes of places and spots under an adjacency relation, so we use two union-find data structures to represent them.

Algorithm 6.1. The following algorithm computes the faces, components, and relations between them.

initialize union-find data structures $F$ for faces and $C$ for components

initialize parent pointer arrays $P F$ for faces and $P C$ for components

for $h=0$ to $D . N$ do

for $k=0$ to $D . W(h)$ do

if $s_{h, k}$ adjacent to $s_{h-1, k}$ and to $s_{h-1, k-D . \Delta(h-1)}$ then $\operatorname{UNION}(F(h-1, k), F(h-1, k-D \cdot \Delta(h-1)))$ $F(h, k) \leftarrow F(h-1, k)$

else if $s_{h, k}$ adjacent to $s_{h-1, k}$ only then $F(h, k) \leftarrow F(h-1, k)$

else if $s_{h, k}$ adjacent to $s_{h-1, k-D . \Delta(h-1)}$ only then $F(h, k) \leftarrow F(h-1, k-D \cdot \Delta(h-1))$

else

$F(h, k) \leftarrow$ a fresh face id

$P F(h, k) \leftarrow(h-1, D \cdot H(h-1))$

\section{end if}

end for

for $k=0$ to $D \cdot W(h)-1$ do

Update components similarly

end for

end for

Unions of faces are performed when scanning vertices with no output. Each of them costs $O\left(\log ^{*} f\right)$, where $f$ is the number of faces of the diagram and $\log ^{*}$ is the log-star function. So total cost of all unions of faces is $O\left(v \log ^{*} f\right)$. Scanning the diagram with the two loops takes $O(v e)$ operations, and checking if two spots or places are adjacent takes constant time. Therefore, the computation of the faces, components and their relations can be done in $O(v e)$.

Then, we apply the algorithm of Theorem 5.8 to compute the right normal form of each component, which can be done again in quadratic time.
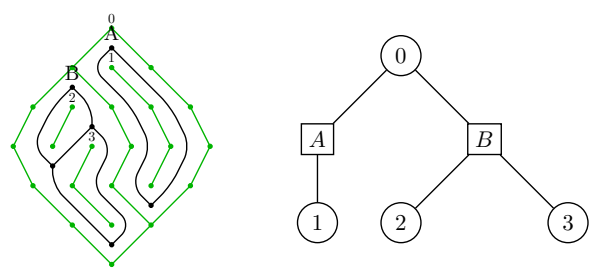

Figure 19. A diagram and its structural tree 
Finally, the structural tree of the diagram is converted to an integer recursively in Algorithm 6.2, where we assume a coding function $\chi$ injectively mapping any tuple of integers to an integer (using an appropriate encoding). As the structural tree is a complete invariant for diagram equivalence, we obtain the following theorem.

A note of caution about this last step is that it does rely pretty crucially on the encoding of large data structures into integers and exploits the fact that the computational model lets us compare unbounded integers for free in constant time, which could be unrealistic for practical purposes. For implementations, this can be mitigated using hashing techniques which let compare large datastructures quickly on average, rejecting different structures quickly and only resorting to full comparison when the hashes are equal.

Algorithm 6.2. The following algorithm recursively computes an integer representation for a structural tree.

if $n$ is a face node then

compute the integer representation of its children components recursively;

sort the list of children components as $l$ return $\chi(l)$

\section{end if}

if $n$ is a component node with normalized root component $c$ then

sort the children faces by order of introduction in the normalized component $c$

compute the integer representation of the children faces recursively as $l$, preserving the order; return $\chi(c, l)$

end if

Theorem 6.3. The word problem for string diagrams in a monoidal category can be solved in $O(v e)$, where $v$ is the number of vertices and $e$ is the number of edges.

\section{LineAR-Time SOLUTiOn TO THE WORD PROBlEM IN THE CONNECTED CASE}

In this section we show how the word problem can be solved in linear time for boundaryconnected diagrams via a reduction to the problem of map isomorphism. In the disconnected case, the components enclosed in a face can spin around each other, so comparing two faces amounts to comparing their sets of components. Therefore, there is little hope to extend this result to the disconnected case.

We first recall some background notions of topological graph theory. We refer the interested reader to [MT01] for a more in-depth treatment of these notions.

7.1. Background on planar maps. A multigraph is a set of vertices $V$ and of edges $E$ where each edge $e \in E$ is associated with a set of one or two vertices $V(e)$. In other words it is an undirected graph where multiple edges can exist between two vertices, and loops are allowed.

A planar map is a discrete representation of the embedding of a connected multigraph (seen as a topological space) in a surface.

Definition 7.1. A map is a set $\Omega$ of darts (or half-edges) and two permutations $x$ and $y$ of $\Omega$ such that $x^{2}=1, x$ has no stationary point, and the permutation group $G$ generated by $x$ and $y$ is transitive (for any $a, b \in \Omega$ there is $g \in G$ such that $g(a)=b$ ). 


$$
\begin{aligned}
& x=\left(\begin{array}{ll}
12 \\
2
\end{array}\right)\left(\begin{array}{ll}
34 \\
4
\end{array}\right)\left(\begin{array}{l}
56 \\
6
\end{array}\right)\left(\begin{array}{l}
78 \\
8
\end{array}\right)(910)
\end{aligned}
$$

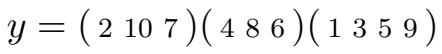

(A) A planar map given by two permutations

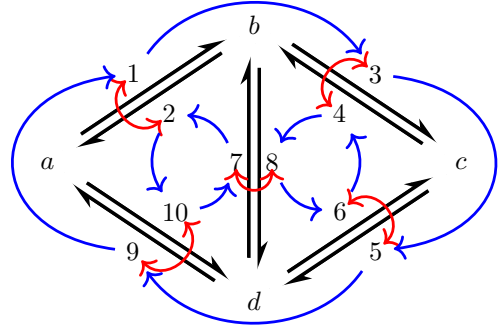

(B) Graphical representation where darts are numbered half-edges

Two maps are isomorphic when there is a bijection between their sets of darts respecting the permutations $x$ and $y$ of both maps.

In a map $m$, the cycles of $x$ are called edges of $m$. The cycles of $y$ are called faces of $m$. The cycles of $x y$ are called vertices. The Euler characteristic of $m$ is

$$
\chi(m)=v-e+f
$$

where $v$ is the number of vertices, $e$ of edges and $f$ of faces. A map $m$ is planar if $\chi(m)=2$.

Any embedding of a multigraph in the plane gives rise to a planar map.

Theorem 7.2 [JS78]. Any two embeddings of a multigraph in the plane are isotopic if and only if the corresponding planar maps are isomorphic.

Theorem 7.3 [HW74]. Determining if two planar maps are isomorphic can be decided in linear time.

Again, the same note of caution about the comparison of unbounded integers in constant time applies to the latter result, but hashing should provide a satisfactory implementation in practice.

Our goal is to reuse this last result to solve the word problem for connected string diagrams. However, the word problems for string diagrams and for planar maps do not match: Figure 21 shows two string diagrams which are isotopic as planar maps but not equivalent as string diagrams.

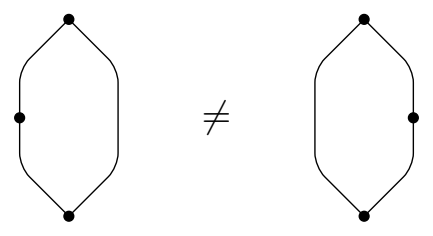

Figure 21. Two non-equivalent string diagrams which are isotopic as maps

7.2. Directed planar maps. Maps are embeddings of undirected multigraphs. In this section, we introduce an analoguous notion for directed multigraphs. A directed multigraph is a set of vertices $V$ and a set of edges $E$, each edge being associated to a pair of vertices $(s, t)$ (its source and target). A directed multigraph is connected if it is connected as an undirected multigraph. 
Definition 7.4. A directed map is a map $(\Omega, x, y)$ together with a choice of distinguished darts $D \subseteq \Omega$ such that exactly one dart in each cycle of $x$ belongs to $D$.

Two directed maps are isomorphic when they are isomorphic as maps and furthermore the bijection respects the distinguished darts. Similarly to Figure 21, there are directed maps which are isomorphic as undirected maps but not as directed maps.

Given a directed planar map $M$, we can define a planar map $\iota(M)$ by replacing each directed edge by an undirected graph which encodes the direction of the original edge:

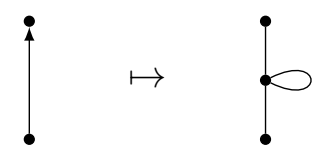

Proposition 7.5. Two directed planar maps $M, M^{\prime}$ are isomorphic if and only if the undirected planar maps $\iota(M)$ and $\iota\left(M^{\prime}\right)$ are isomorphic.

Proof. If $M$ and $M^{\prime}$ as isomorphic then clearly so are $\iota(M)$ and $\iota\left(M^{\prime}\right)$. Conversely, assume that $\iota(M)$ and $\iota\left(M^{\prime}\right)$ are isomorphic maps via an isomorphism $\phi$. Say that a vertex $v \in \iota(M)$ is a loop root if a loop is rooted on $v$. Given the definition of $\iota$, the image $\iota(u)$ of a vertex $u \in M$ cannot be a loop root, as any loop on $u$ in $M$ is translated to non-loop edges in $\iota(M)$. Therefore, there is a bijection between the loop roots of $\iota(M)$ and the edges of $M$. As loop roots are preserved by graph isomorphism, $\phi$ induces a bijection between the loop roots of $\iota(M)$ and $\iota\left(M^{\prime}\right)$, so we have a bijection $\psi$ between the edges of $M$ and $M^{\prime}$. This bijection in turn determines a directed graph isomorphism between $M$ and $M^{\prime}$. For instance the source vertex of an edge can be recovered from its loop root $u$ : follow the edge which comes after the loop, when browsing incident edges of $u$ in clockwise order. Similarily the target vertex can be recovered. Finally, as $\phi$ is a map isomorphism, the cyclic order of edges around vertices is preserved, so $\psi$ is a directed map isomorphism between $M$ and $M^{\prime}$.

Corollary 7.6. Testing whether two acyclic directed planar maps are isomorphic can be done in linear time.

Proof. The translation via $\iota$ can be computed in linear time so the problem reduces to deciding undirected planar map isomorphism, which is linear by Theorem 7.3.

Proposition 7.7. Two embeddings of connected directed multigraphs in the plane are isotopic if and only if the corresponding directed maps are isomorphic.

7.3. From string diagrams to maps. We translate any string diagram $D$ to a directed planar map $\gamma(D)$ by replacing each vertex by the gadget below. The original edges coming from $D$ inherit their orientation from the string diagram (top to bottom), and we add two dangling edges for each vertex. These additional dangling edges are useful for vertices with only inputs or only outputs by blocking any cyclic permutation of these edges around the vertex. ${ }^{5}$

\footnotetext{
${ }^{5}$ These dangling edges are only useful for vertices with only inputs or only outputs but we choose to add them to all vertices for the sake of uniformity.
} 

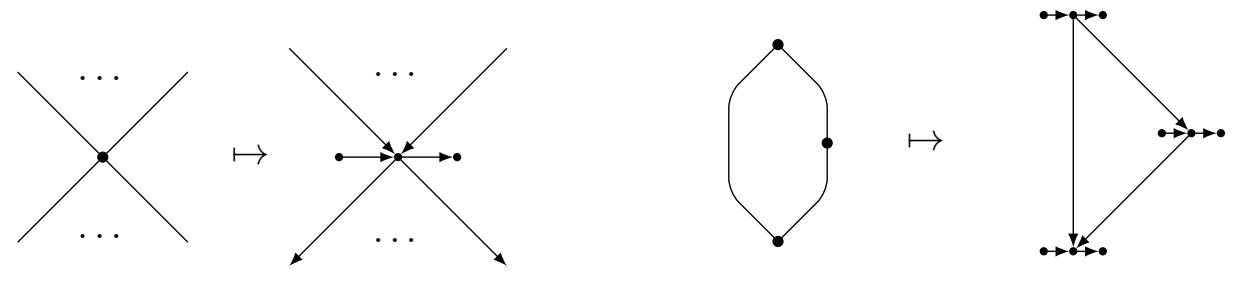

Figure 22. Translation of a string diagram to a directed map.

Theorem 7.8. Any two connected diagrams are equivalent if and only if the induced directed maps are isomorphic.

Proof. Exchanges on connected diagrams preserve the translation to directed maps so any two equivalent connected diagrams are mapped to isomorphic directed maps. For the converse direction, we can therefore assume that the two diagrams $D, D^{\prime}$ are in right normal form. Let $\phi$ be the map isomorphism between the corresponding directed maps $\gamma(D), \gamma\left(D^{\prime}\right)$. First, $\gamma(D)$ and $\gamma\left(D^{\prime}\right)$ have the same number of vertices and so do $D$ and $D^{\prime}$. Call $n$ the number of vertices of $D$.

We prove by induction on $n$ that $D=D^{\prime}$. We reuse the induction technique introduced in Section 5: diagram $D$ contains a leaf or a face.

If $D$ contains a leaf $l$, this leaf is mapped to a vertex $\gamma(l)$ connected to three edges. As $\phi(\gamma(l))$ is also connected to three edges, there is a leaf $l^{\prime} \in D^{\prime}$ such that $\gamma\left(l^{\prime}\right)=\phi(\gamma(l))$. Because $\phi$ is an isomorphism of directed maps, the orientations of $l$ and $l^{\prime}$ are the same: they are both single-input or both single-output vertices. Moreover, they are connected to their parent vertices at the same position in their list of inputs or outputs, thanks to the auxilliary edges added in the translation. Consider the diagrams $E$ and $E^{\prime}$ obtained from $D$ and $D^{\prime}$ by removing $l$ and $l^{\prime}$ respectively. These diagrams are in right normal form. The isomorphism $\phi$ induces a map isomorphism between $\gamma(E)$ and $\gamma\left(E^{\prime}\right)$ so by induction $E=E^{\prime}$. By Lemma 5.1, $D=D^{\prime}$.

If $D$ contains a face $f$, this face is mapped to a face $\gamma(f)$ in $\gamma(D)$. The face $\phi(\gamma(f))$ is itself the image of a face $f^{\prime} \in D^{\prime}$. Because $\phi$ preserves edge orientations, the mountain ranges of $f$ and $f^{\prime}$ are equal. Let $e$ be an eliminable edge in $f$ and let $e^{\prime}$ be the preimage of $\phi(\gamma(e))$ in $f^{\prime}$. By equality of the mountain ranges, $e^{\prime}$ is also eliminable in $f^{\prime}$. By Lemma 5.7, removing $e$ from $D$ and $e^{\prime}$ from $D^{\prime}$ gives diagrams $F$ and $F^{\prime}$ both in right normal form. Again we can apply the induction hypothesis to $F$ and $F^{\prime}$, so $F=F^{\prime}$, and therefore $D=D^{\prime}$.

Corollary 7.9. The word problem for connected string diagrams can be solved in linear time.

Proof. The translation $\gamma$ from string diagrams to directed planar maps can be computed in linear time. The decision problem therefore reduces to the word problem for acyclic directed planar maps, which is solvable in linear time by Corollary 7.6.

\section{RECUMBEnt isotopy}

Joyal and Street's theorem relating diagram deformations to the axioms of monoidal categories (Theorem 1.4) requires the deformations to be recumbent. This means that at each stage of the deformation, the diagram's edges must remain upright, as shown in Figure 23. It 


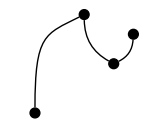

(A) A recumbent plane diagram

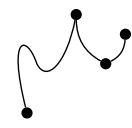

(в) A locally recumbent plane diagram

FiguRE 23. Examples of topological diagrams.

was conjectured by Selinger [Sel11] that the recumbency condition can be weakened. For this weakening, the requirement that all wires must flow vertically can be dropped, but we must keep the requirement that wires stay connected to their endpoints from the same side. Figure 24 shows a counter-example for the conjecture without this last condition.

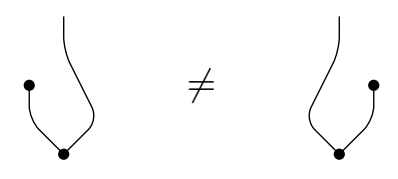

FiguRE 24. Arbitrary planar isomorphism does not respect morphism equality.

We now show how our reduction from string diagrams to planar maps can be used to prove Selinger's conjecture, generalizing Joyal and Street's Theorem 1.4. To extend this result to disconnected diagrams, we only need to extend the notion of directed map to disconnected cases.

Definition 8.1. A disconnected planar map is defined recursively as a tree, as follows.

- A face node is a set of component nodes (possibly empty).

- A component node is a planar map $m$, an outer face $f_{0} \in m$ and face nodes for each face $f \neq f_{0}$ of $m$.

A disconnected planar map is given by its root face node, which has finite depth.

As this definition mirrors that of the structural tree of a diagram (Definition A.18), it is straightforward to extend the translation of Section 7.3 to translate any diagram $D$ to a disconnected planar map.

Equivalence of disconnected planar maps is defined by pointwise equivalence of the planar maps involved. By completeness of the structural tree for string diagrams (Theorem A.21), two string diagrams are equivalent if and only if the corresponding disconnected planar maps are equivalent. For this reason, Theorem 7.2 can be extended to the disconnected case: two disconnected planar maps are equivalent if and only if their embeddings in the plane are isotopic. We therefore obtain the following theorem:

Theorem 8.2. Two string diagrams are equivalent if and only if their translations as disconnected planar maps from Section 7 are isotopic.

This generalizes Joyal and Street's result in the way hinted by Selinger's conjecture: the isotopy is unconstrained, although some gadgets have been added to enforce the preservation of the order of inputs and outputs around vertices.

This generalization has a clean statement in terms of planar pivotal monoidal categories [Sel11, Section 4.2]. The coherence theorem for their graphical calculus is stated as follows [Sel11, Theorem 4.14]. 
Theorem 8.3 Coherence for pivotal categories. A well-formed equation between morphisms in the language of pivotal categories follows from the axioms of pivotal categories if and only if it holds in the graphical language up to planar isotopy.

We write monoidal signature for the generating data for a monoidal category which is free on objects and morphisms, and given a monoidal signature $\Sigma$, we write $\mathrm{M}(\Sigma)$ for the free monoidal category on $\Sigma$, and $\mathrm{P}(\Sigma)$ for the free pivotal category on $\Sigma$. Combining Theorems 8.2 and 8.3 then yields the following.

Corollary 8.4. Given a monoidal signature $\Sigma$, the obvious embedding functor $F: \mathrm{M}(\Sigma) \rightarrow$ $\mathrm{P}(\Sigma)$ is faithful.

Proof. For $A, B \in \mathrm{Ob}(\mathrm{M}(\Sigma))$ and morphisms $f, g: A \rightarrow B$, Theorem 8.3 says that $F(f)=$ $F(g)$ just when the string diagrams for $f$ and $g$ are isotopic. But then by Theorem 8.2, we also have $f=g$, and hence faithfulness.

8.1. Acknowledgements. We thank the members of the Séminaire de catégories supérieures, polygraphes et homotopie at IRIF, Paris for their feedback on these results, in particular Simon Forest and Samuel Mimram, and we are also grateful to Vincent Vidal, Éric Colin de Verdière and Arnaud de Mesmay for their feedback. Jamie Vicary acknowledges funding from the Royal Society and Antonin Delpeuch is supported by an EPSRC scholarship.

\section{REFERENCES}

[Bac14] Miriam Backens. The ZX-calculus is complete for stabilizer quantum mechanics. New Journal of Physics, 16(9):093021, 2014. doi:10.1088/1367-2630/16/9/093021.

[BKV16] Krzysztof Bar, Aleks Kissinger, and Jamie Vicary. Globular: an online proof assistant for higherdimensional rewriting. In Leibniz International Proceedings in Informatics, volume 52, pages 34:1-34:11, 2016. ncatlab.org/nlab/show/Globular.

[Bur93] Albert Burroni. Higher-dimensional word problems with applications to equational logic. Theoretical Computer Science, 115(1):43-62, July 1993. doi:10.1016/0304-3975(93)90054-W.

[BV] Krzysztof Bar and Jamie Vicary. Data structures for quasistrict higher categories. In Proceedings of LICS $201 \%$.

[BV16] Krzysztof Bar and Jamie Vicary. Data structures for quasistrict higher categories. arXiv:1610.06908 [math], October 2016. arXiv:1610.06908.

[CCS08] Stephen Clark, Bob Coecke, and Mehrnoosh Sadrzadeh. A compositional distributional model of meaning. In Proceedings of the Second Quantum Interaction Symposium (QI-2008), pages 133-140, 2008.

[dd13] Éric Colin de Verdière and Arnaud de Mesmay. Testing Graph Isotopy on Surfaces. arXiv:1310.2745 [cs, math], October 2013. arXiv:1310.2745.

[DDK10] Lucas Dixon, Ross Duncan, and Aleks Kissinger. Open Graphs and Computational Reasoning. Electronic Proceedings in Theoretical Computer Science, 26:169-180, June 2010. doi:10.4204/ EPTCS.26.16.

[DL14] Ross Duncan and Maxime Lucas. Verifying the Steane code with Quantomatic. Electronic Proceedings in Theoretical Computer Science, 171:33-49, December 2014. doi:10.4204/EPTCS. 171. 4.

[FM18] Simon Forest and Samuel Mimram. Coherence of Gray categories via rewriting. page 17, 2018.

[GHWZ16] Neil Ghani, Jules Hedges, Viktor Winschel, and Philipp Zahn. Compositional game theory. arXiv:1603.04641 [cs], March 2016. arXiv:1603.04641.

[GJL17] Dan R. Ghica, Achim Jung, and Aliaume Lopez. Diagrammatic Semantics for Digital Circuits. arXiv:1703.10247 [cs], March 2017. arXiv:1703.10247. 
[GM18] Yves Guiraud and Philippe Malbos. Polygraphs of finite derivation type. Mathematical Structures in Computer Science, 28(02):155-201, February 2018. doi:10.1017/S0960129516000220.

[HHV19] Lukas Heidemann, Nick Hu, and Jamie Vicary. Homotopy.io. 2019. doi:10.5281/zenodo. 2540764.

[Hot65] Guenter Hotz. Eine Algebraisierung des Syntheseproblems von Schaltkreisen I. Elektronische Informationsverarbeitung und Kybernetik, 1(3):185-205, 1965.

[HW74] J. E. Hopcroft and J. K. Wong. Linear Time Algorithm for Isomorphism of Planar Graphs (Preliminary Report). In Proceedings of the Sixth Annual ACM Symposium on Theory of Computing, STOC '74, pages 172-184, New York, NY, USA, 1974. ACM. doi:10.1145/800119.803896.

[JS78] Gareth A. Jones and David Singerman. Theory of Maps on Orientable Surfaces. Proceedings of the London Mathematical Society, s3-37(2):273-307, September 1978. doi:10.1112/plms/s3-37. 2.273.

[JS88] André Joyal and Ross Street. Planar diagrams and tensor algebra. Unpublished manuscript, available from Ross Street's website, September 1988.

[JS91] André Joyal and Ross Street. The geometry of tensor calculus, I. Advances in Mathematics, 88(1):55-112, 1991.

[Laf03] Yves Lafont. Towards an algebraic theory of Boolean circuits. Journal of Pure and Applied Algebra, 184(2-3):257-310, November 2003. doi:10.1016/S0022-4049(03)00069-0.

[Laf09] Yves Lafont. Diagram rewriting and operads. page 14, 2009.

[Mak05] M. Makkai. The word problem for computads. May 2005.

[Mel12] P. Melliès. Game Semantics in String Diagrams. In 2012 27th Annual IEEE Symposium on Logic in Computer Science, pages 481-490, June 2012. doi:10.1109/LICS.2012.58.

[Mim14] Samuel Mimram. Towards 3-Dimensional Rewriting Theory. Logical Methods in Computer Science, 10(2), April 2014. arXiv:1403.4094, doi:10.2168/LMCS-10(2:1) 2014.

[ML63] Saunders Mac Lane. Natural associativity and commutativity. Rice Univ. Studies, 49(4):28-46, 1963.

[MT01] Bojan Mohar and Carsten Thomassen. Graphs on Surfaces, volume 10. JHU Press, 2001.

[Sel11] Peter Selinger. A survey of graphical languages for monoidal categories. In New Structures for Physics, volume 813 of Lecture Notes in Physics, pages 289-233. Springer, 2011.

\section{Appendix A. Extension to DisconneCted diagrams}

As the transformation described in Figure 15 is still applicable to disconnected diagrams, we consider a diagram $D$ without input or output edges $(D \cdot S=0$ and $D \cdot W(D \cdot N)=0)$.

Definition A.1. For each level $h \in[D . N+1]$, we define symbols $\left(s_{h, k}\right)_{0 \leq k \leq D . N(h)}$. The symbol $s_{h, k}$ is the spot at height $h$ and position $k$, which represents the empty space between the $k$ th and $k+1$ th edge at level $h$ (including diagram boundaries for the extrema). Similarly, we define symbols $\left(p_{h, k}\right)_{1 \leq k \leq D . N(h)}$. The symbol $p_{h, k}$ represents the intersection of the $k$ th edge that crosses level $h$ and level $h$ itself: we call this a place. Vertices of the diagram are places too, and we represent the vertex between heights $h$ and $h+1$ as $v_{h}$.

Definition A.2. Two spots $s_{h, k}$ and $s_{h+1, k^{\prime}}$ are adjacent when either $k=k^{\prime}$ and $D . H(h) \geq k$ or $k+D \cdot \Delta(h)=k^{\prime}$ and D.H(h) $+D \cdot I(h) \leq k$.

Graphically, two spots are adjacent if they lie in neighbouring slices and they are in the same region of the diagram seen as a planar graph. We will formally define this notion of region by taking the connected closure of this adjacency relation.

Definition A.3. A face is a connected component of spots for the adjacency relation defined above.

Definition A.4. Two places $p_{h, k}$ and $p_{h+1, k^{\prime}}$ at consecutive levels are adjacent if they are on the same edge. Formally, this happens when either $k=k^{\prime}$ and $D \cdot H(h)>k$, 
or $k+D \cdot \Delta(h)=k^{\prime}$ and D.H(h) + D.I $(h) \leq k . D \cdot H(h) \leq k<D . H(h)+D \cdot I(h)$ and $D . H(h) \leq k^{\prime}<D . H(h)+D . O(h)$. A place $p_{h, k}$ and a vertex $v_{h}^{\prime}$ are adjacent when Two places $p_{h, k}, p_{h, k^{\prime}}$ at the same level are adjacent if D.H(h) $\leq k, k^{\prime}<D . H(h)+D . I(h)$ or $D \cdot H(h-1) \leq k, k^{\prime}<D \cdot H(h-1)+D \cdot O(h-1)$.

Definition A.5. A component is a connected component of places for the adjacency relation defined above.

Lemma A.6. Any exchange $D \rightarrow D^{\prime}$ induces bijections $\phi_{F}$ and $\phi_{C}$ between the faces and components of $D$ and $D^{\prime}$.

Proof. Let $n$ and $n+1$ be the levels exchanged. By symmetry we can assume it is a right exchange. Let us define $\phi_{C}$ by mapping each spot in $D$ to a spot in $D^{\prime}$, such that the adjacency relation is respected. Let $s_{h, k}$ be a spot. If $h \leq n$ or $h>n+1$ (the spot lies in a slice that is untouched by the exchange) then $\phi_{C}\left(s_{h, k}\right)=s_{h, k}$. Otherwise, $h=n+1$. If $k \leq D \cdot H(n+1)$ (the spot lies to the left of both nodes exchanged) then $\phi_{C}\left(s_{n+1, k}\right)=s_{h, k}$ again. If $k>D \cdot H(n)+D \cdot I(n)$ (the spot lies to the right of both nodes exchanged) then $\phi_{C}\left(s_{n+1, k}\right)=s_{h, k-D . \Delta(n)+D . \Delta(n+1)}$. If $k>D \cdot H(n+1)$ and $k<D \cdot H(n+1)+D \cdot I(n+1)$ (the spot lies in one of the input branches of the node at height $n+1)$ then $\phi_{C}\left(s_{n+1, k}\right)=$ $s_{n, k}$ (the spot just above). Similarly if $k>D \cdot H(n)$ and $k<D \cdot H(n)+D \cdot O(n)$ then $\phi_{C}\left(s_{n+1, k}\right)=s_{n+2, k+D . \Delta(n+1)}$ (the spot just below). Finally, if $k \geq D \cdot H(n+1)+D \cdot I(n+1)$ and $k \leq D . H(n)$ then $\left.\phi_{C}\left(s_{n+1, k}\right)=s_{n, k-D . \Delta(n)+D \cdot \Delta(n+1)}\right)$. In each of these cases one can check that $\phi_{C}$ preserves the adjacency relationship for spots. The mapping for places $\phi_{F}$ can be defined similarly.

Definition A.7. Given a level $h$, a spot $s_{h, k}$ and a place $p_{h, k^{\prime}}$ are neighbours if $k=k^{\prime}$ or $k+1=k^{\prime}$. Furthermore, spots $s_{h, 0}$ and $s_{h, D . W(h)}$ are neighbours of the boundary.

Lemma A.8. There is a unique face containing spots which are neighbours of the boundary. We denote it by $f_{0}$.

Proof. Any neighbour of the boundary is adjacent to the neighbours of the boundary above and below it. By assumption, there is only one spot at the source and target levels. Therefore, all neighbours of the boundary are connected together.

Definition A.9. A component $c$ neighbours a face $f$ when there is a place $p \in c$ neighbouring a spot $s \in f$. We denote it by $c \diamond f$.

Neighbourhood is preserved by exchanges, in the following sense:

Lemma A.10. Let $d, d^{\prime}$ be diagrams where $d^{\prime}$ is obtained from d by exchanges. The bijections between faces and components of $d$ and $d^{\prime}$ induced by the exchanges respect the neighbourhood relation.

Proof. It suffices to show that any neighbourhood relation that holds between slices affected by an exchange also holds in the exchanged diagram. This can be achieved by simple inspection of the definition of neighbourhood and adjacency on spots and places.

Definition A.11. Given a level $h$, two spots $s_{h, k}$ and $s_{h, k^{\prime}}$ are over-connected if they are connected by a path of spots which never go below level $h$. Similarly, over-connectivity is defined for places too.

Note that over-connectivity is an equivalence relation on spots at the same level, and similarly for places. 


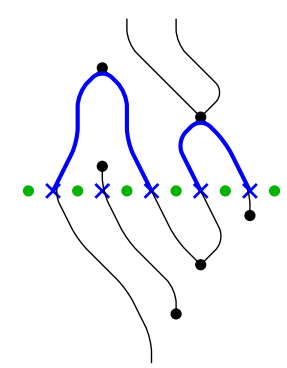

Figure 25. Over-connectivity on an example

Lemma A.12. Given a level $h$, assume that two distinct spots $s_{h, k}$ and $s_{h, k^{\prime}}$ are overconnected. Furthermore, assume that $\forall k<i<k^{\prime}, s_{h, i}$ is over-connected to neither $s_{h, k}$ nor $s_{h, k^{\prime}}$. Then the places $p_{h, k}$ and $p_{h, k^{\prime}-1}$ are over-connected.

Proof. By induction on the distance $h$ from the top of the diagram. The property holds trivially for the topmost slice as no two spots are over-connected at this level.

Assuming it holds at level $h$ and consider spots $s_{h+1, k}$ and $s_{h+1, k^{\prime}}$ over-connected. If they are both connected to the same spot $s_{h, k}$ then all places between them are over-connected, so the result holds. Otherwise, they are connected to different spots $s_{h, k_{0}}$ and $s_{h, k_{0}^{\prime}}$ respectively which are over-connected. Let $s_{h, i_{1}}, \ldots, s_{h, i_{q}}$ be the spots between $s_{h, k_{0}}$ and $s_{h, k_{0}^{\prime}}$ which are over-connected to $s_{h, k_{0}}$ and $s_{h, k_{0}^{\prime}}$. Each of these spots are not connected to any spot at level $h+1$, so the places neighbouring them are all connected via the same morphism between level $h$ and $h+1$. In particular, $p_{h, i_{1}-1}$ and $p_{h, i_{q}}$ are neighbours.

Apply the induction hypothesis to the pairs $\left(s_{h, k_{0}}, s_{h, i_{1}}\right)$ and $\left(s_{h, i_{q}}, s_{h, k_{0}^{\prime}}\right): p_{h, k_{0}}$ and $p_{h, i_{1}-1}$ are over-connected and so are $p_{h, i_{q}}$ and $p_{h, k_{0}^{\prime}-1}$. Therefore $p_{h, k_{0}}$ and $p_{h, k_{0}^{\prime}-1}$ are over-connected.

Lemma A.13. Let $f$ be a face, $f \neq f_{0}$. There is a component $c$ such that for each level $h$ containing a spot in $f$, the place to the left of the first spot in $f$ at level $h$ and the place to the right of the last spot in $f$ at level $h$ are both in $c$. Such a component $c$ is therefore unique. We say that $c$ encloses $f$, denoted by $f \prec c$.

Proof. First, consider the highest level $h_{0}$ where $f$ occurs. All spots in $f$ at $h_{0}$ are not connected to any spot at the higher level, so their neighbouring places are all connected to the morphism above. Let $c$ be their common component. For any further level $h$ we prove the result by induction. Consider the first and last spots $s_{h, k}, s_{h, k^{\prime}}$ which belong to $f$ at level $h$. We show that $p_{h, k-1}$ belongs to $c$. Showing that so does $p_{h, k^{\prime}}$ is similar. Let $s_{h-1, k_{0}}$ be the leftmost spot neighboured by $s_{h, k}$ at level $h-1$. If $s_{h-1}$ is the first spot $s_{h, k_{0}}$ in $f$ at $h-1$, then by induction $p_{h-1, k_{0}-1}$ belongs to $c$ and is connected to $p_{h, k-1}$, so $p_{h, k-1}$ belongs to $c$. Otherwise, let $s_{h-1, i_{1}}, \ldots, s_{h-1, i_{p}}$ be the spots in $f$ to the left of $s_{h-1, k_{0}}$. We can apply Lemma A.12 to $s_{h-1, i_{p}}$ and obtain that $p_{h-1, i_{p}}$ and $p_{h-1, k_{0}-1}$ are connected. Furthermore, the $s_{h-1, i_{1}}, \ldots s_{h-1, i_{p}}$ are not neighbours of any spots at level $h$, so the edges separating them are all connected to the same vertex between $h$ and $h-1$. So $p_{h-1, i_{p}}$ and $p_{h-1, i_{1}-1}$ are neighbours, and finally $p_{h-1, k_{0}-1}$ and $p_{h-1, i_{1}-1}$ are connected. By induction, $p_{h-1, i_{1}-1}$ belongs to $c$, so so does $p_{h-1, k_{0}-1}$.

Definition A.14. Given a face $f$, a spot $s \in f$ is maximal if it is at the highest level where spots of $f$ occur. Similarly, it is minimal if it is at the lowest level where spots of $f$ occur. 
Note that a face can have multiple maximal or minimal spots.

Lemma A.15. Maximal and minimal spots in a face only neighbour the enclosing component, or the boundary in the case of the root face.

Proof. This is a direct consequence of the proof of Lemma A.13.

Definition A.16. Let $f$ be a face. For each component $c$ neighbour of $f$ such that $c$ does not enclose $f$, we say that $f$ encloses $c$, denoted by $c \prec f$.

The enclosure relation is preserved by exchanges as follows:

Lemma A.17. $\phi_{F}$ and $\phi_{C}$ respect $\prec$, i.e. $f \prec c \Leftrightarrow \phi_{F}(f) \prec \phi_{C}(c)$ and $c \prec f \Leftrightarrow \phi_{C}(c) \prec$ $\phi_{F}(f)$.

Proof. By Lemma A.10 and because $c \prec f \Leftrightarrow c \diamond f \wedge \neg(f \prec c)$, it is enough to show preservation of $f \prec c$.

Let $d \rightarrow d^{\prime}$ be a right exchange, $f$ be a face in $d$ enclosed by $c$. Let $s_{h} \in f$ be a maximal spot in $f$, and $s_{l} \in f$ be a minimal spot in $f$. If $s_{h}$ is untouched by the exchange, then it is still maximal in $\phi_{F}(f)$, and $\phi_{C}(c)$ is still the only component neighboured by $\phi_{F}(f)$ at $s_{h}$ 's slice, so we have $\phi_{F}(f) \prec \phi_{C}(c)$. Similarly, if $s_{l}$ is untouched by the exchange, $\phi_{F}(f) \prec \phi_{C}(c)$. If both $s_{l}$ and $s_{h}$ are touched by the exchange, then they are equal in $d$ and $f$ neighbours only $c$ in $d$. By Lemma A.10, $\phi_{F}(f)$ neighbours only $\phi_{C}(c)$. As $\phi_{F}(f)$ is not the root face in $d^{\prime}, \phi_{F}(f) \prec \phi_{C}(c)$.

We next introduce an order on the faces enclosed by a component $c$. Let $N(c)$ be the right normal form of $c$, seen as a standalone diagram. The right reduction from $c$ to $N(c)$ induces a bijection between the faces of $c$ and those of $N(c)$.

Given two faces $f, f^{\prime}$ in $N(c)$, consider the leftmost maximal spots $s_{h, k}, s_{h^{\prime}, k^{\prime}}$ of $f$ and $f^{\prime}$. We order $f$ and $f^{\prime}$ by lexicographic order on the pairs $(h, k),\left(h^{\prime}, k^{\prime}\right)$. This defines an order $<$ on faces of $N(c)$ and therefore on faces of $c$.

Definition A.18. We inductively define the structural tree of faces and components. Given a face $f, T(f)=\{T(c) \mid c$ component enclosed by $f\}$. Given a component $c$, let $f_{1}, \ldots, f_{n}$ be the set of faces enclosed by $c$, ordered with the order defined above. We set $T(c)=$ $\left(N(c), T\left(f_{1}\right), \ldots, T\left(f_{n}\right)\right)$. Finally, the structural tree $T(D)$ of the entire diagram is $T\left(f_{0}\right)$.

To make sure that this tree is finite, we must make sure that none of its nodes is a child of itself.

Lemma A.19. $\prec$ is well-founded.

Proof. A diagram contains a finite number of components and faces. It is therefore enough to show that given a component $c$, it is impossible that $c \prec \cdots \prec c$. We first show that if $c \prec f \prec c^{\prime}$, then at each level $h$ where $f$ appears, then for any place $p_{h, k} \in c$ there are places $p_{h, a}, p_{h, b} \in c^{\prime}$ with $a<k<b$. This is a simple consequence of Lemma A.13. Then, by induction, we extend this to the transitive closure of $\prec$, which shows the result.

Lemma A.20. $T(D)$ is invariant under exchanges.

Proof. By Lemma A.17, $\prec$ is invariant under exchanges. The order on the faces enclosed by a given component is also invariant as it is defined on the right normal form of the component. 
A.1. Completeness of the structural tree. We show that the structural tree $T(D)$ of a diagram is a complete invariant for exchanges:

Theorem A.21. Two diagrams $D, D^{\prime}$ with no source and target edges are equivalent if and only if $T(D)=T\left(D^{\prime}\right)$.

To prove this theorem we introduce a few notions of diagram surgery, to manipulate components and faces.

Definition A.22. Given a diagram $D$ and a spot $s \in D$, the injection of a closed diagram $D^{\prime}$ at $s$, denoted by $I_{s}^{D}\left(D^{\prime}\right)$, is obtained by inserting $D^{\prime}$ in place of $s$ in $D$. Concretely, this means that the vertices of $D^{\prime}$ are inserted at the slice of $s$, shifted to the right by the number of edges to the left of $s$. Formally, $I_{s}^{D}\left(D^{\prime}\right)$ is defined as follows:

$$
\begin{aligned}
& I_{s}^{D}\left(D^{\prime}\right) \cdot S=D \cdot S \\
& I_{s}^{D}\left(D^{\prime}\right) \cdot N=D \cdot N+D^{\prime} \cdot N \\
& I_{s}^{D}\left(D^{\prime}\right) \cdot H(h)= \begin{cases}D \cdot H(h) & \text { if } h<a \\
D^{\prime} \cdot H(h-a)+k & \text { if } a \leq h<b \\
D \cdot H\left(h-D^{\prime} \cdot N\right) & \text { if } b \leq h\end{cases} \\
& I_{s}^{D}\left(D^{\prime}\right) \cdot I(h)= \begin{cases}D \cdot I(h) & \text { if } h<a \\
D^{\prime} \cdot I(h-a) & \text { if } a \leq h<b \\
D \cdot I\left(h-D^{\prime} \cdot N\right) & \text { if } b \leq h\end{cases} \\
& I_{s}^{D}\left(D^{\prime}\right) \cdot O(h)= \begin{cases}D \cdot O(h) & \text { if } h<a \\
D^{\prime} \cdot O(h-a) & \text { if } a \leq h<b \\
D \cdot O\left(h-D^{\prime} \cdot N\right) & \text { if } b \leq h\end{cases}
\end{aligned}
$$

where $s=s_{a, k}$ and $b=a+D^{\prime} . N$.

By abuse of language, if $x \in D^{\prime}$ is a place, spot, face or component, then we denote again by $x$ the corresponding place, spot, face or component in $I_{s}^{D}\left(D^{\prime}\right)$, as this is unambiguously defined.

Lemma A.23. Let $D$ be a diagram and $s, s^{\prime} \in D$ be spots in the same face $f \in D$. For all closed diagram $D^{\prime}, I_{s}^{D}\left(D^{\prime}\right) \simeq I_{s^{\prime}}^{D}\left(D^{\prime}\right)$.

Proof. Let us assume $s$ and $s^{\prime}$ are adjacent. Let $v$ be the vertex between the two slices containing $s$ and $s^{\prime}$. The vertices of $D^{\prime}$ in $I_{s}\left(D^{\prime}\right)$ can be successively exchanged with $v$, leading to $I_{s^{\prime}}^{D}\left(D^{\prime}\right)$, which shows that $I_{s}^{D}\left(D^{\prime}\right) \simeq I_{s^{\prime}}^{D}\left(D^{\prime}\right)$. By induction, this can be repeated for any adjacency path between two spots in the same face.

Bearing in mind that this is only defined up to exchange, we can therefore write $I_{f}^{D}\left(D^{\prime}\right)$ to inject $D^{\prime}$ anywhere in the face $f$.

Lemma A.24. Injection respects exchanges on the outer and inner diagrams: If $D \simeq D^{\prime}$ and $C \simeq C^{\prime}$, then for any face $f \in D$ and its corresponding face $f^{\prime} \in D^{\prime}, I_{f}^{D}(C) \simeq I_{f^{\prime}}^{D^{\prime}}\left(C^{\prime}\right)$.

Proof. Any exchange $C \rightarrow_{R} C^{\prime}$ translates into a single exchange $I_{f}^{D}(C) \rightarrow_{R} I_{f}^{D}\left(C^{\prime}\right)$. So, by induction, if $C \simeq C^{\prime}$, then $I_{f}^{D}(C) \simeq I_{f}^{D}\left(C^{\prime}\right)$. To show that injection respects equivalence on the outer diagram, let $s \in f$ and consider a single rewriting step $D \rightarrow_{R} D^{\prime}$. If $s$ is not 
in the slice between the two vertices $u$ and $v$ being exchanged in $D$, then it corresponds to a spot $s^{\prime} \in D^{\prime}$. We have $s^{\prime} \in f^{\prime}$ and $I_{s}^{D}(C) \rightarrow_{R} I_{s^{\prime}}^{D^{\prime}}(C)$ in one step again. Otherwise, $D^{\prime} . N$ exchanges are required to move $u$ past $D^{\prime}$, one to exchange $u$ and $v$, and $D^{\prime} . N$ again to move $v$ past $D^{\prime}$. So $I_{s}^{D}(C) \simeq I_{s}^{D^{\prime}}(C)$. So injections are compatible with exchanges both on the inner and outer diagram.

Definition A.25. Let $D$ be a diagram and $c \in D$ be a component. The erasure of $c$ in $D$, denoted by $D-c$, is the diagram obtained by removing from $D$ any vertex from $c$ or its sub-components.

Lemma A.26. Let $D$ be a diagram and $c \in D$ be an acyclic component. Then there is a face $f \in D-c$ such that $D \simeq I_{f}^{D-c}(c)$.

Proof. Pick a vertex $r \in c$ : we will consider $c$ as a tree rooted in $r$. By induction on this tree, we are going to gather all vertices around $r$, meaning that the heights of these vertices in the diagram form an interval.

Say that a vertex $v \in c$ is collapsed if the set of diagram heights of the vertices in its subtree form an interval. For any $v \in c$, we show that $D$ is equivalent to a diagram $D^{\prime}$ where $v$ is collapsed and such that the vertical order of vertices which are outside this subtree is preserved in $D^{\prime}$.

If $v$ is a leaf, it is always collapsed. Consider the case where $v$ has children $u_{1}, \ldots, u_{n}$. Because $c$ is acyclic, it is possible to exchange each child $u_{i}$ with any vertex on a slice between $u_{i}$ and $v$, so we can assume that the vertical positions of $v$ and $u_{1}, \ldots, u_{n}$ form an interval. By induction, each $u_{i}$ can be successively collapsed without changing the vertical order of vertices which are not in the subtree of $u_{i}$. Once this is done, the vertical position of vertices in the subtrees of the $u_{i}$ and $v$ form an interval, so $v$ is collapsed. By doing so we have preserved the vertical ordering of vertices outside the subtree of $v$.

Therefore, there is a $D^{\prime}$ where $r$ is collapsed. Let $c^{\prime}$ be the component corresponding to $c$ in $D^{\prime}$. Let $f^{\prime} \in D^{\prime}$ be the face enclosing $c^{\prime}$ in $D^{\prime}$. We have $I_{f^{\prime}}^{D^{\prime}-c^{\prime}}\left(c^{\prime}\right)=D^{\prime}$. By invariance

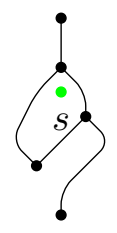

(A) The diagram $D$ with a spot $s \in D$
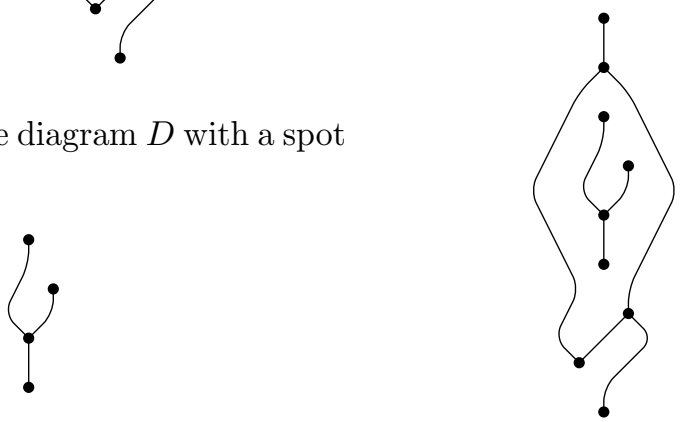

(в) The diagram $D^{\prime}$

(c) The diagram $I_{s}^{D}\left(D^{\prime}\right)$

FiguRE 26. Injection of a diagram in a face 
of injection up to exchanges (Lemma A.24), $I_{f^{\prime}}^{D^{\prime}-c^{\prime}}\left(c^{\prime}\right) \simeq I_{f}^{D-c}(c)$ for the corresponding face $f \in D-c$.

Definition A.27. Let $D$ be a diagram and $f \in D$ be a face that does not neighbour the boundary. The erasure of $f$ in $D$, denoted by $D-f$, is the diagram obtained by removing all spots in $f$ and descendant faces. Formally, let $P(h, i, j)=\left|\left\{s_{h, k} \mid i \leq k<j, s_{h, k} \in f^{\prime} \prec * f\right\}\right|$. Then $D-f$ is defined as follows:

$$
\begin{aligned}
& (D-f) . S=D . S \\
& (D-f) \cdot N=D \cdot N \\
& (D-f) \cdot H(h)=D \cdot H(h)-P(h, 0, D \cdot H(h)+1) \\
& (D-f) \cdot I(h)=\left\{\begin{array}{l}
1 \text { if } D \cdot I(h)=0 \text { and } P(h, D \cdot H(h), D \cdot H(h))=1 \\
D \cdot I(h)-P(h, D \cdot H(h)+1, D \cdot H(h)+D \cdot I(h)+1) \text { otherwise }
\end{array}\right. \\
& (D-f) . O(h)=\left\{\begin{array}{l}
1 \text { if } D \cdot O(h)=0 \text { and } P(h+1, D \cdot H(h), D \cdot H(h)+1)=1 \\
D \cdot O(h)-P(h+1, D \cdot H(h)+1, D \cdot H(h)+D \cdot O(h)+1) \text { otherwise }
\end{array}\right.
\end{aligned}
$$

One can check that this defines a valid diagam.

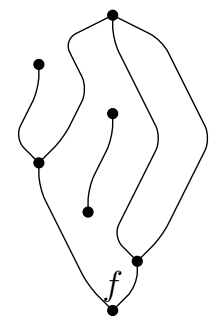

(A) A face $f$ in diagram $D$

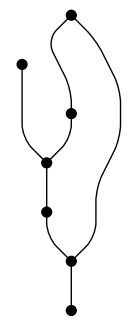

(B) The diagram $D-f$

Lemma A.28. Let $s \in D$ be a spot. Then for any closed diagram $c$ and face $f \in c$, $I_{s}^{D}(c-f)=I_{s}^{D}(c)-f$.

Proof. This can be checked directly from the definitions of injections and erasures.

Lemma A.29. Let $D$ be a diagram and $f \in D$ be a face. For any diagram $D^{\prime} \simeq D-f$, there is a diagram $D_{0} \simeq D$ such that $D^{\prime}=D_{0}-f$.

Proof. By inspection of the definition of $D-f$ one can see that any exchange on $D-f$ can be lifted back to an exchange at the same height on $D$ (the converse is false). By induction, this defines $D_{0}$.

Lemma A.30. Let $D$ be a diagram and $c \in D$ be an arbitrary component (not necessarily acyclic this time). Then there is a spot $s \in D-c$ such that $D \simeq I_{s}^{D-c}(c)$. 
Proof. Let $f_{1}, \ldots, f_{k}$ be all the faces enclosed by $c$. Consider $D^{\prime}=D-f_{1} \cdots-f_{k}$. Let $c^{\prime}$ be the component corresponding to $c$ in $D^{\prime}$ : as a diagram, $c^{\prime}=c-f_{1} \cdots-f_{k}$. As $c^{\prime}$ does not enclose any face, $c^{\prime}$ is acyclic. By Lemma A.26, we can gather $c^{\prime}$ in one spot: there is $s \in D^{\prime}-c^{\prime}$ such that $D^{\prime} \simeq I_{s}^{D^{\prime}-c^{\prime}}\left(c^{\prime}\right)$. (In fact, because all the faces removed from $D$ to obtain $D^{\prime}$ are enclosed by $c, D^{\prime}-c^{\prime}=D-c$.) Then, by Lemma A.29, there is a $D_{0} \simeq D$ such that $D_{0}-f_{1} \cdots-f_{k}=I_{s}^{D^{\prime}-c^{\prime}}\left(c^{\prime}\right)$. By Lemma A.28, $I_{s}^{D^{\prime}-c^{\prime}}\left(c^{\prime}\right)=I_{s}^{D^{\prime}-c^{\prime}}\left(c-f_{1} \cdots-f_{k}\right)=$ $I_{s}^{D^{\prime}-c^{\prime}}(c)-f_{1} \cdots-f_{k}$. Therefore, we obtain $D_{0}-f_{1} \cdots-f_{k}=I_{s}(c)-f_{1} \cdots-f_{k}$ and finally $D_{0}=I_{s}^{D^{\prime}-c^{\prime}}(c)=I_{s}^{D-c}(c)$.

We can now prove Theorem A.21, showing the completeness of the structural tree for exchanges.

Proof. Let $C, D$ be diagrams such that $T(C)=T(D)$.

To each node $n$ of $T(C)$ we can associate diagrams $C_{n}$ (respectively $D_{n}$ ) obtained by erasing vertices not contained in the subtree below $n$ in $C$ (respectively $D$ ). We show by induction on $n$ that $C_{n} \simeq D_{n}$.

If $n$ is a leaf face node, then both $C_{n}$ and $D_{n}$ are empty diagrams and are therefore equivalent. If $n$ is a leaf component node, then both $C_{n}$ and $D_{n}$ are acyclic connected diagrams with identical right normal forms, so they are equivalent.

If $n$ is an internal face node, let $\left\{c_{1}, \ldots, c_{m}\right\}$ be its child components. By induction their corresponding diagrams in $C$ and $D$ are pairwise equivalent. We can apply Lemma A.30 for each of them and express both $C$ and $D$ as iterated injections of the $c_{i}$ in the empty diagram: therefore $C_{n} \simeq D_{n}$.

If $n$ is an internal component node, let $\left(f_{1}, \ldots, f_{m}\right)$ be its child faces. Again, by induction their corresponding diagrams in $C$ and $D$ are pairwise equivalent. Moreover, the components corresponding to $n$ in $C$ and $D$ have the same right normal form $F$. We can therefore obtain both $C$ and $D$ as iterated injections of the $f_{i}$ in the faces of $F$, in the designated order. Therefore $C_{n} \simeq D_{n}$, which completes the proof. 